\title{
Cdk5 drives formation of heterogeneous pancreatic neuroendocrine tumors
}

Angela M. Carter $\mathbb{D}^{1 凶}$, Nilesh Kumar ${ }^{2}$, Brendon Herring ${ }^{1}{ }^{1}$, Chunfeng Tan $^{3}$, Rachael Guenter ${ }^{1}$, Rahul Telange ${ }^{1}$, Wayne Howse ${ }^{1}$, Fabrice Viol ${ }^{4}$, Tyler R. McCaw ${ }^{1}$, Hayden H. Bickerton ${ }^{5,6}$, Priyanka Gupta ${ }^{1}$, Frank Gillardon ${ }^{7}$, Eugene A. Woltering ${ }^{8}$, Deepti Dhall ${ }^{9}$, John Totenhagen ${ }^{10}$, Ronadip R. Banerjee $\mathbb{I D}^{11}$, Elizabeth M. Kurian ${ }^{12}$, Sushanth Reddy ${ }^{1,13}$, Herbert Chen ${ }^{1,13}$, Joerg Schrader ${ }^{4}$, J. Bart Rose ${ }^{1,13}$, M. Shahid Mukhtar ${ }^{1,2}$ and James A. Bibb (D) ${ }^{1,13 凶}$

(c) The Author(s) 2021

Pancreatic neuroendocrine tumors (PanNETs) are a heterogeneous population of neoplasms that arise from hormone-secreting islet cells of the pancreas and have increased markedly in incidence over the past four decades. Non-functional PanNETs, which occur more frequently than hormone-secreting tumors, are often not diagnosed until later stages of tumor development and have poorer prognoses. Development of successful therapeutics for PanNETs has been slow, partially due to a lack of diverse animal models for pre-clinical testing. Here, we report development of an inducible, conditional mouse model of PanNETs by using a bi-transgenic system for regulated expression of the aberrant activator of Cdk5, p25, specifically in $\beta$-islet cells. This model produces a heterogeneous population of PanNETs that includes a subgroup of well-differentiated, non-functional tumors. Production of these tumors demonstrates the causative potential of aberrantly active Cdk5 for generation of PanNETs. Further, we show that human PanNETs express Cdk5 pathway components, are dependent on Cdk5 for growth, and share genetic and transcriptional overlap with the INS-p25OE model. The utility of this model is enhanced by the ability to form tumor-derived allografts. This new model of PanNETs will facilitate molecular delineation of Cdk5-dependent PanNETs and the development of new targeted therapeutics.

Oncogenesis (2021)10:83; https://doi.org/10.1038/s41389-021-00372-5

\section{INTRODUCTION}

Pancreatic neuroendocrine tumors (PanNETs) are a diverse group of neoplasms that originate from islet cells of the pancreas [1]. These tumors have the potential to secrete a range of bioactive hormones such as insulin, glucagon, and somatostatin. Tumors that secrete quantities of hormones that result in elevations in blood plasma levels are classified as functional [2]. Functional tumors produce hormonal syndromes commensurate with the hormone produced in excess [3]. Functional tumors are typically lower in grade and have good prognoses, possibly due to early detection as a result of the syndromes experienced by patients [4]. However, the majority of PanNETs are non-functional and on average have comparatively worse prognoses [5]. Historically rare, the incidence of PanNETs in the United States increased 8-fold from 1973 to 2012 [4]. Surgical resection provides excellent outcomes and long-term survival for patients with early stage primary tumors [6-8]. However, many PanNETs are metastatic at diagnosis and there are no curative therapies for advanced disease $[9,10]$.
Multiple molecular alterations have been implicated in the development of PanNETs. Mutations in the gene MEN1 occur in $\sim 40 \%$ of PanNET patients and changes in DAXX/ATRX are present in another $40 \%$. Roughly $15 \%$ of patients possess changes that target the mTOR pathway, including mutations in TSC2, PIK3CA, or PTEN [11-13]. Unfortunately, thus far, no correlation has been observed between the presence of these mutations and patient response to specific pathway-targeted therapies in NET clinical trials [14]. Recently, cyclin-dependent kinase 5 (Cdk5) was implicated in the growth of several types of neuroendocrine tumors including PanNETs [15-17]. Interestingly, the presence of a set of downstream biomarkers of Cdk5 pathway activation was predictive of tumor growth inhibition in preclinical testing of a Cdk5-targeted therapy [15].

Cdk5 is a non-canonical member of the Cdk family of prolinedirected serine/threonine kinases [18]. Traditional family members, such as Cdk1, 2, 4, and 6, are important cell cycle regulators that are activated by cyclins and required for cell division [19]. Unlike these family members, Cdk5 is not activated by cyclins and is not

\footnotetext{
${ }^{1}$ Department of Surgery, University of Alabama at Birmingham, Birmingham, AL 35233, USA. ${ }^{2}$ Department of Biology, University of Alabama at Birmingham, Birmingham, AL 35233, USA. ${ }^{3}$ Department of Psychiatry, University of Texas Southwestern Medical Center, Dallas, TX 75390, USA. ${ }^{4}$ Department of Internal Medicine, University Hospital HamburgEppendorf, Hamburg 20246, Germany. ${ }^{5}$ Department of Medicine, University of Alabama at Birmingham, Birmingham, AL 35233, USA. ${ }^{6}$ UAB Comprehensive Diabetes Center, University of Alabama at Birmingham, Birmingham, AL 35233, USA. ${ }^{7}$ Boehringer Ingelheim Pharma GmbH \& Co. KG, CNS Diseases Research, Birkendorferstrasse 65, 88397 Biberach an der Riss, Germany. ${ }^{8}$ Department of Surgery, Louisiana State University Health Sciences Center, New Orleans, LA 70112, USA. ${ }^{9}$ Department of Anatomic Pathology, University of Alabama at Birmingham, Birmingham, AL 35233, USA. ${ }^{10}$ Department of Radiology, University of Alabama at Birmingham, Birmingham, AL 35233, USA. ${ }^{11}$ Department of Medicine, Division of Endocrinology, Diabetees \& Metabolism, Johs Hopkins school of Medicine, Baltimore, MD 21224, USA. ${ }^{12}$ Department of Pathology, University of Texas Southwestern Medical Center, Dallas, TX 75390, USA. ${ }^{13} \mathrm{O}$ 'Neal Comprehensive Cancer Center, University of Alabama at Birmingham, Birmingham, AL 35233, USA.

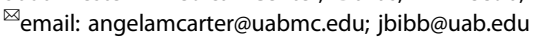


required for normal cell division. Instead, Cdk5 is regulated through binding to cofactors p35 or p39 $[20,21]$. The resulting protein complex plays a prominent role in several physiological processes in neuronal cells, such as proper migration for CNS development and function [22, 23]. Interestingly, aberrant activation of Cdk5 has been implicated in several neurodegenerative diseases [20]. The pathological role of Cdk5 is facilitated through calpain cleavage of p35 to p25, a highly stable fragment that exhibits mislocalization in cells but retains the ability to bind and activate Cdk5 [24, 25]. Cdk5 pathway components are also expressed in neuroendocrine cells of pancreatic islets where they contribute to normal hormone secretion and $\beta$-cell survival [2630]. New studies show that under conditions of aberrant activation in non-neuronal cells, Cdk5 can hijack signaling components traditionally involved in the cell cycle and successfully promote proliferation and/or migration [16, 31-36]. Here, we show that Cdk5 and its activators are retained in islet cells that develop into PanNETs in humans and that aberrant activation of Cdk5 is involved in human PanNET cell growth. Furthermore, we show the potential for Cdk5 to drive development of PanNETs by demonstrating that expression of the aberrant activator, p25, in islets of mice, initiates tumor formation. Importantly, these PanNETs exhibit a heterogeneous phenotype that includes both functional and non-functional, well-differentiated tumors.

\section{RESULTS}

To better understand the relevance of the Cdk5 pathway to human PanNETs, we performed immunostaining on distinct groups of grade 1 human tumors for Cdk5 pathway components. This revealed the presence of Cdk5 and its activators, p35 and/or p25 (p35/p25) (Fig. 1A) in both functional and non-functional tumors. To gain further insight into the prevalence of these signaling proteins in the PanNET patient population, we performed immunostaining on a PanNET tissue microarray (TMA) composed of 23 grade 1 tumors, 13 grade 2 tumors, 1 grade 3 tumor, and 5 different normal tissue controls. (Fig. 1B, C and Supplementary Table S1). Semi-quantitation revealed clear expression of Cdk5 and p35/p25 throughout these grades of tumors (Fig. 1D, E) and elevated expression relative to a normal placenta control sample.

To determine if $\mathrm{Cdk} 5$ and its activators play a functional role in PanNETs, we next examined a set of human PanNET-derived cell lines including the well-established BON and QGP lines, and three newly derived lines NT-3, NT-18P, and NT-18LM [37]. These cell lines expressed Cdk5 and its aberrant activator, p25 (Fig. 1F). We previously found that growth of the pancreatic carcinoid cell line, BON, was blocked by 4 different selective Cdk5 inhibitors and not by Cdk2 and Cdk4 specific inhibitors [15]. Here, we show that growth of all four PanNET cell lines tested is inhibited by the Cdk5selective inhibitor, IndoA (Fig. 1G-K). These data indicate that Cdk5 dependence is a common feature shared by many PanNETs.

Cdk5 has also been suggested to play a role in growth and migration of pancreatic ductal adenocarcinoma (PDAC) [32, 38]. Therefore, as a comparison, we confirmed expression of Cdk5 in two different human PDAC cell lines, Panc1 and MiaPaCa, and tested these lines for growth inhibition in response to IndoA (Supplementary Fig. S1). Interestingly, the $\mathrm{IC}_{50}$ for MiaPaCa, a line in which Cdk5 inhibition induces Caspase 3/7 activity [39], was within the range of responsiveness of the PanNET cell lines, while the $I C_{50}$ for Panc1 trended higher than all other lines and was significantly higher than QGP (Fig. 1K). These data agree with a growing body of information indicating that Cdk5 may be an alternative driver of growth and/or survival in a range of cancer types [18].

To determine if Cdk5 has the potential to behave as a causative factor in PanNET tumorigenesis, we generated a bi-transgenic mouse line in which expression of the aberrant Cdk5 activator, p25, can be selectively induced in $\beta$-cells of the pancreas by addition of the small molecule doxycycline (dox) to drinking water. This was achieved by crossing the Ins2-rtTA mouse line, [40] that expresses the reverse tetracycline transactivator under the control of the insulin promoter, with the tetOp-p25GFP line, [41] that expresses p25GFP under the control of the tetOp promoter, (Fig. 2A) to produce bi-transgenic offspring (INS-p25OE). As previously observed with some doxycycline, dox inducible systems, a low level of transgene expression was observed in the absence of dox. However, administration of $1 \mathrm{~g} / \mathrm{L}$ dox to INSp25OE animals for 4-8 weeks further induced expression of the p25-GFP transgene in pancreatic islets (Fig. 2B, C). Formation of solid lesions in the pancreas were observed as early as 6 months post-induction of p25GFP expression (Fig. 2D and Supplementary Fig. S2). As confirmation that transgene expression does not occur ubiquitously throughout tissues of these animals, we examined samples of pancreatic masses along with liver and kidney tissues for p25GFP expression after 12 months of dox administration and found no evidence of p25GFP expression in non-pancreatic tissues (Fig. 2E).

Histological analysis of these masses showed a "nesting" pattern in cellular architecture that is characteristic of PanNETs (Fig. 2F). Immunoblot and immunostain confirmed the presence of p25GFP and $\mathrm{Cdk} 5$ in the lesions (Fig. 2E, G). Furthermore, immunostaining demonstrated the presence of chromogranin A (ChA), confirming the neuroendocrine phenotype of the lesions. Insulin staining verified the masses were composed of $\beta$-cells. In addition, pathological review diagnosed the lesions as well-differentiated PanNETs. These data demonstrate that aberrant activation of the Cdk5 pathway is sufficient to directly promote the formation of PanNETs.

To assess growth rate of the INS-p25OE PanNETs, MRI was performed on tumor-bearing mice over a 20-week period beginning when tumors were $\sim 50 \mathrm{~mm}^{3}$ (Fig. $2 \mathrm{H}$ ). PanNETs in this model exhibited a multiphasic growth pattern. Initial growth was linear with tumors from males and females increasing 3.2-fold and 4.7-fold in size, respectively, over a 5 -week timeline (Fig. $2 \mathrm{l}, \mathrm{J}$ ). This phase was followed by deceleration and an eventual plateau around $400 \mathrm{~mm}^{3}$ (Fig. 2l). Removal of dox, to decrease expression of p25GFP after tumor onset, greatly reduced tumor growth rate (Fig. $2 \mathrm{~J}$ ).

The presence of a linear growth phase allows detection of changes in tumor growth, in response to experimental therapeutics, in smaller cohorts of animals. To further assess the utility of this model for pre-clinical testing, we examined tumors for the presence of somatostatin receptor 2 (SSTR2), a cell-surface protein commonly overexpressed in human PanNETs and targeted by various FDA-approved treatments for PanNETs. All PanNETs tested exhibited clear SSTR2 expression (Fig. 2K).

Human PanNETs present clinically as a highly heterogeneous population of tumors $[1,3]$. Subgroups of tumors secrete a variety of islet derived hormones while others exhibit no detectable hormone production. To characterize the tumors generated in the new INS-p25OE model, we stained sections of fixed tumors for insulin, glucagon, and somatostatin; three hormones commonly expressed in functional human PanNETs. All PanNETs examined expressed insulin in the tumor mass and a few also exhibited expression of glucagon and somatostatin (Fig. 3A).

For a tumor to be definitively categorized as clinically functional, in addition to the presence of the hormone in tumor tissue, circulating blood hormones must be elevated to levels capable of inducing physiological effects. Therefore, plasma samples from animals harboring PanNETs and transgene (-) littermates, as controls, were analyzed for insulin, glucagon, and somatostatin. Tumor-bearing animals were not found to possess statistically higher average levels of any islet hormone analyzed when assessed collectively (Fig. 3B, C).

For higher stringency for classification as non-functional, the data was analyzed again using two standard deviations above the 
A

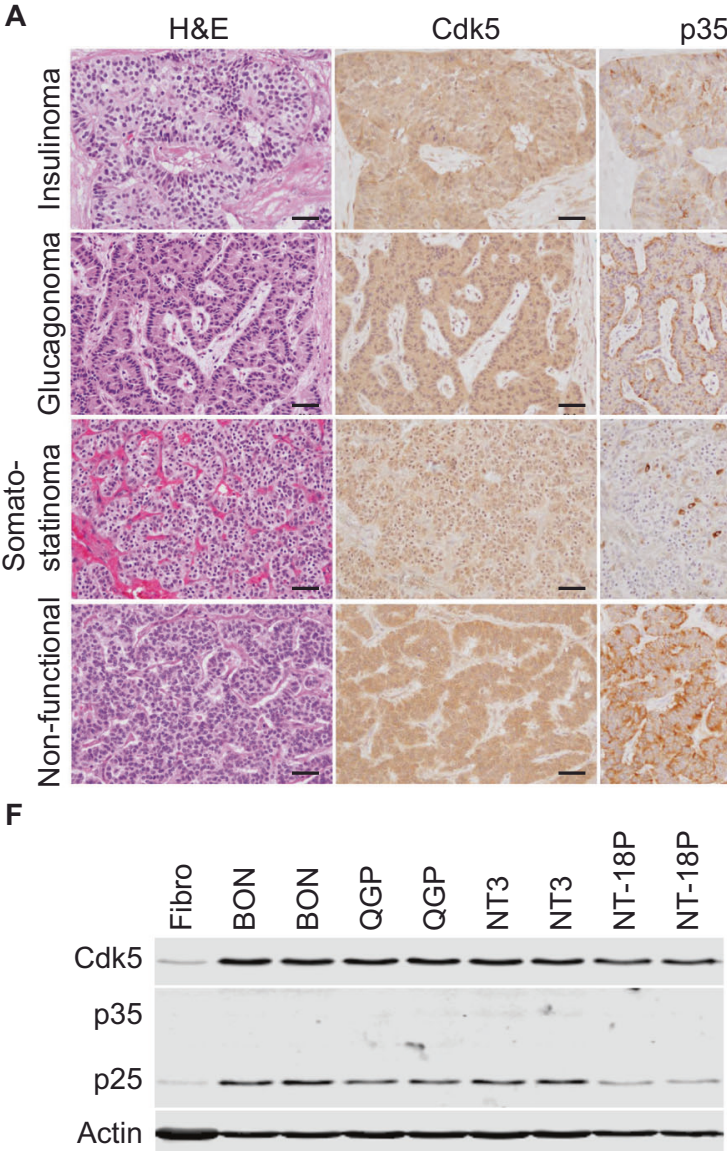

I
B
C

p35/p25
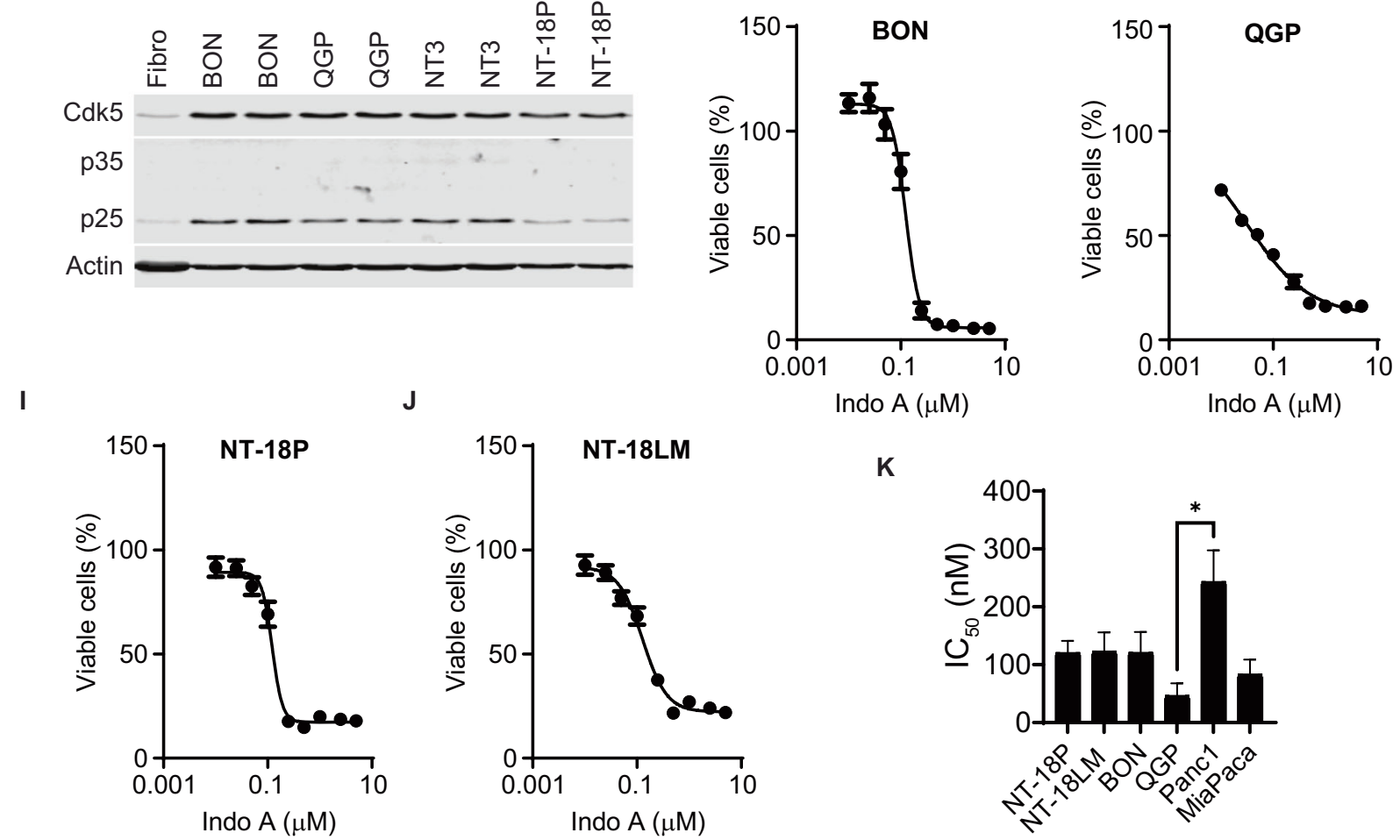

Fig. 1 Human PanNETs are dependent on Cdk5. A H\&E stain and immunostains for Cdk5 and p35/p25 in G1 PanNETs. Scale bars $=50 \mu m$. Immunostains for Cdk5 (B) and p35/25 (C) from human PanNET TMA. Scale bar $=2 \mathrm{~mm}$. Semi-quantitation of Cdk5 expression from B (D) and p35/25 expression from $\mathbf{C}(\mathbf{E})$ normalized to expression levels of each in a normal placenta core; column 1 row 4 of the TMA. Map of TMA in Supplementary Table S1. F Immunoblot of Cdk5 pathway components in fibroblasts and PanNET cells. G-J PanNET cell lines were treated with increasing concentrations of Indo A and monitored for effects on cell viability $(n=3)$. $\mathbf{K} I C_{50}$ values obtained from viability assays in $\mathbf{G}-\mathbf{J}$ and Supplementary Fig. S1; ANOVA with Tukey's multiple comparisons test for $\mathrm{IC}_{50}$ values. All error bars represent SEM.

mean of the control group as the cut-off for normal hormone levels. The average insulin levels in normal females and males was statistically different at 0.6 and $1.1 \mathrm{ng} / \mathrm{mL}$, respectively (Fig. 3B, C) $(p=0.03$ ). Elevations in insulin were present in $57 \%$ (8 of 14 , red symbols) of tumor-bearing females with 10.8-fold being the highest observed increase relative to control animals. Insulin levels were elevated in $41 \%$ ( 7 of 17 , red symbols) of males with 8.9 -fold being the highest elevation observed. Normal glucagon levels for females and males were 125 and $110 \mathrm{pg} / \mathrm{mL}$, respectively. Of tumor-bearing animals, only one male exhibited a 2.3-fold elevation of plasma glucagon, less than $1 \%$ of the total population and within the natural expected Gaussian distribution. Somatostatin levels in control females and males were 15 and $18 \mathrm{pg} / \mathrm{mL}$, respectively. Both normal and tumor-bearing populations of males 
A

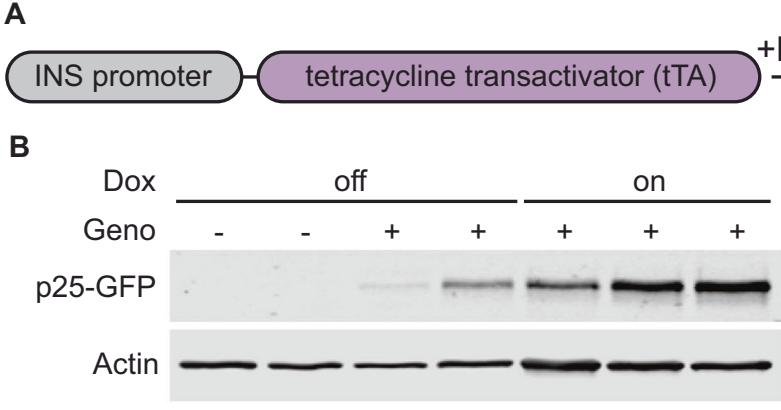

D

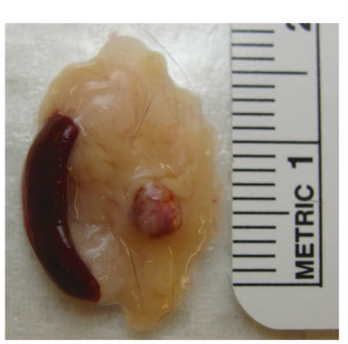

G

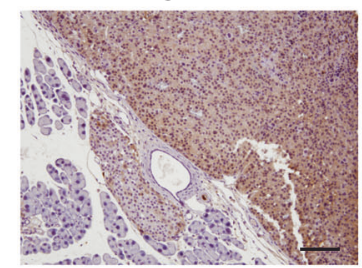

H

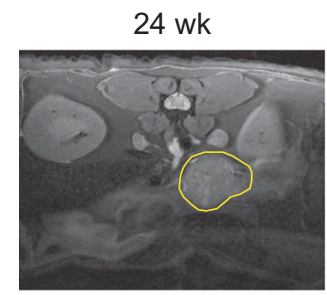

J

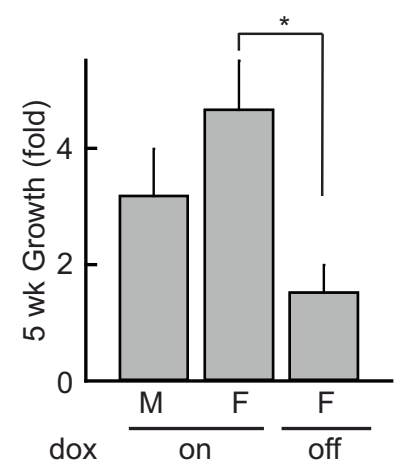

E

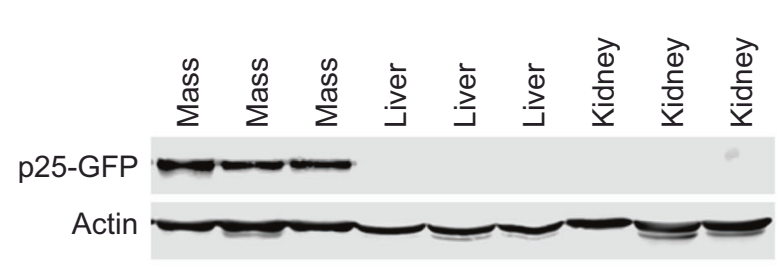

ChA
p25-GFP

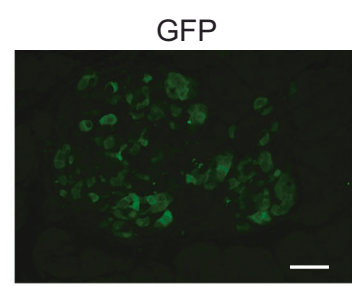

F

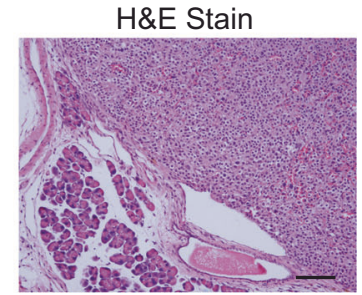

Insulin

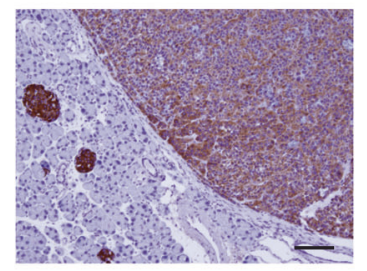

I

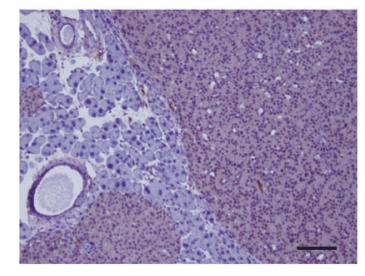

29 wk

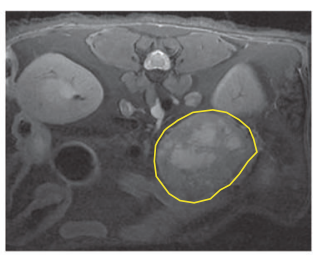

K

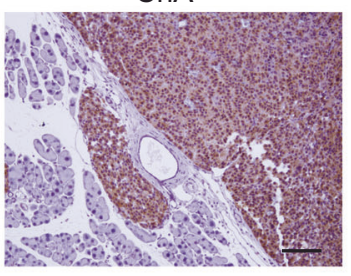

$43 \mathrm{wk}$
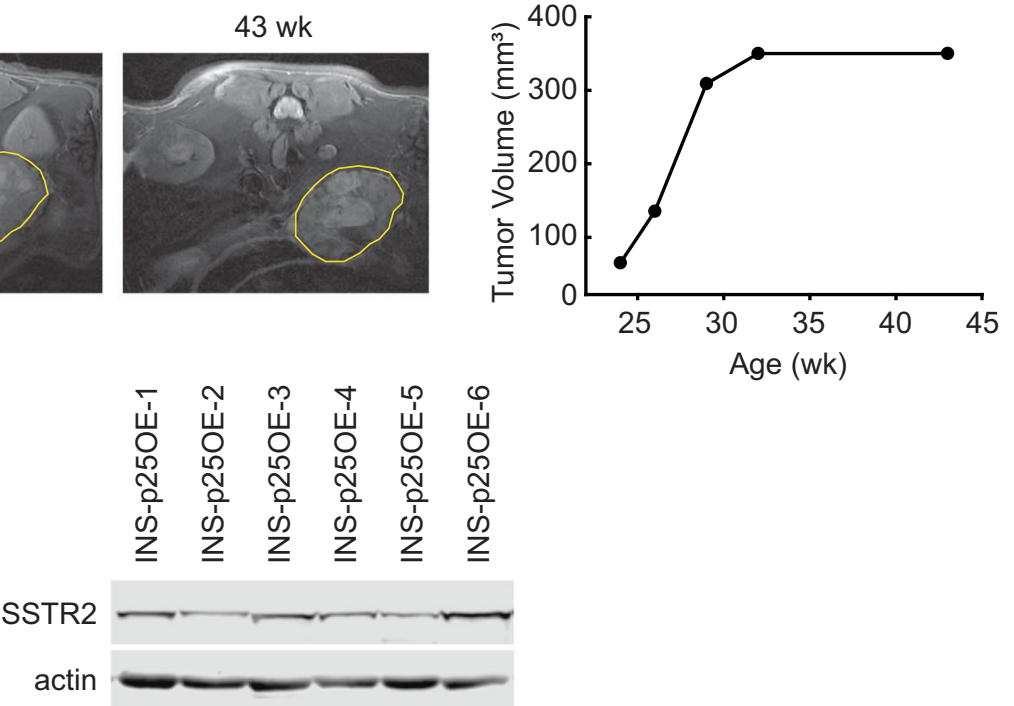

Fig. 2 Aberrant activation of Cdk5 generates PanNETs in an inducible bi-transgenic mouse model. A Schematic of genetic system for regulated tissue-specific expression of p25-GFP. B Immunoblot for expression of p25GFP in islets isolated from transgene negative ( - ) and INS-p25OE (+) mice with (on) or without (off) administration of $1 \mathrm{~g} / \mathrm{L}$ Dox for 4-5 weeks. C H\&E stain and immunofluorescence of sections of pancreas from INS-p25OE mice at 4 weeks post-p25-GFP induction. D Representative gross image of a pancreatic mass from INS-p25OE mice. E Immunoblot for expression of p25-GFP in pancreatic mass, liver, and kidney at 12 months induction. H\&E stain (F), and immunostains (G) of primary PanNET from an INS-25OE animal. Scale bars $=100 \mu \mathrm{m}$. H Axial MRI sections from a representative INS-p25OE mouse. PanNET circumscribed in yellow. I Quantitation of tumor volume over time from a representative INS-p25OE mouse. J Tumor growth, normalized to initial volume, during the linear growth phase; males $(\mathrm{M} ; n=4)$ and females ( $\mathrm{F} ; n=3$ for each group) administered dox since weaning (on) or dox since weaning followed by discontinuation for 5 weeks after initial tumor detection (off). On and off dox only compared within female groups. $\mathbf{K}$ Immunoblot for expression of SSTR2 and actin in INS-p25OE tumors. 
A
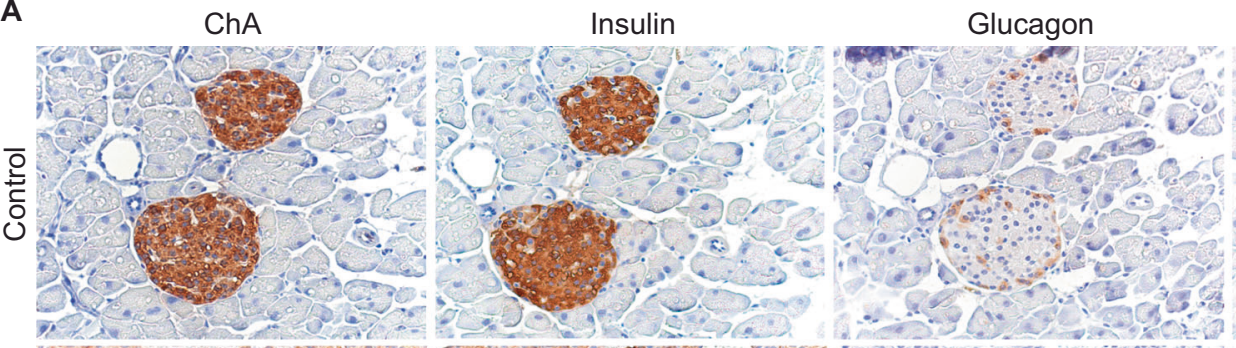

Somatostatin
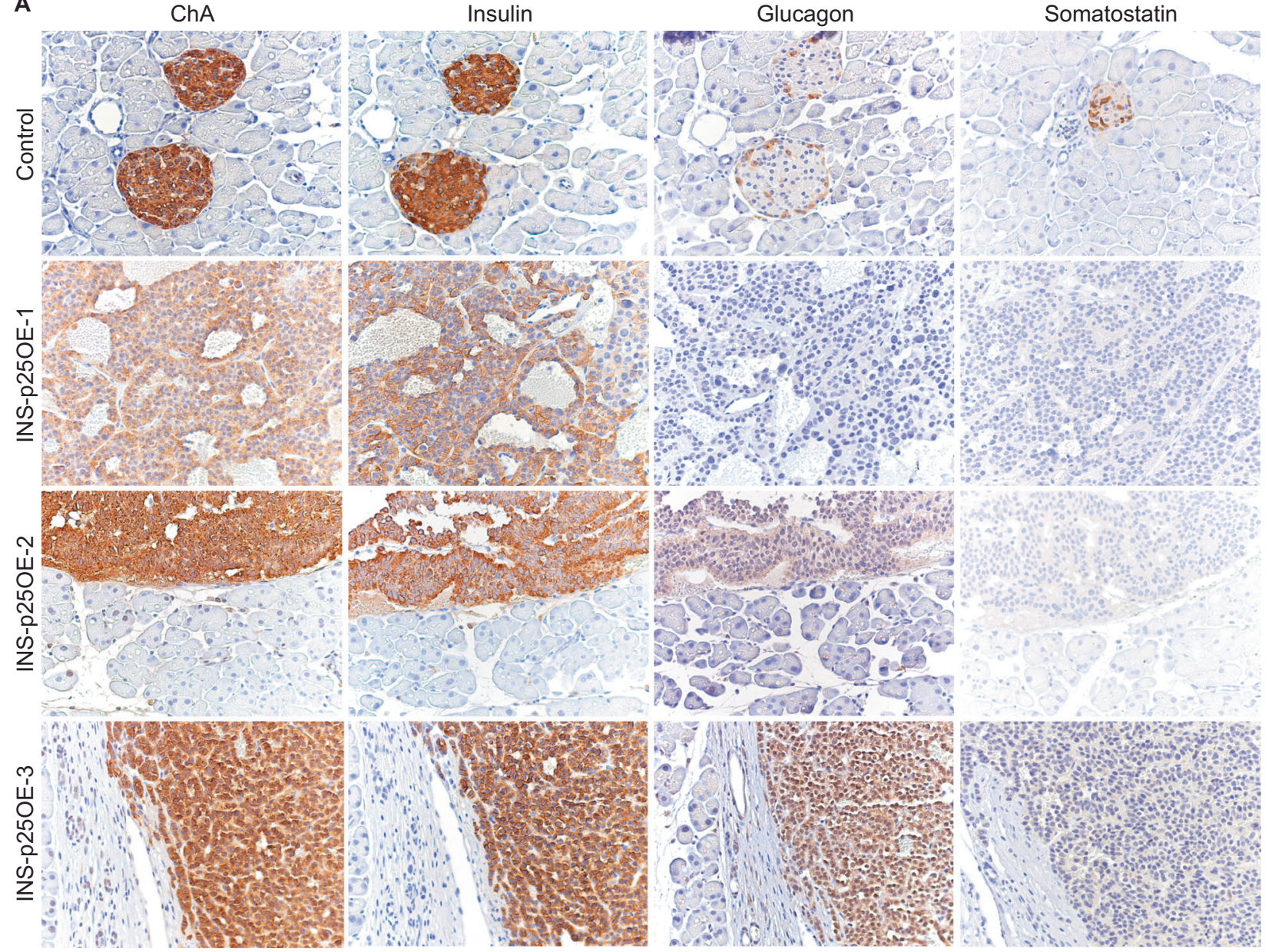

$$
\text { B }
$$

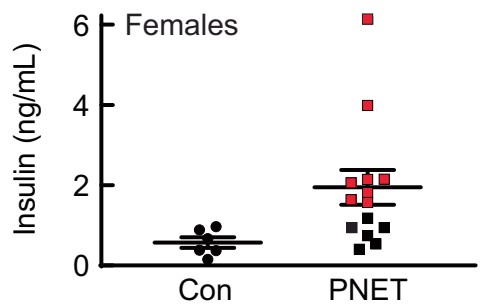

C

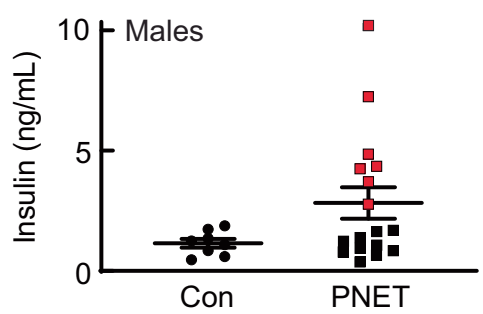

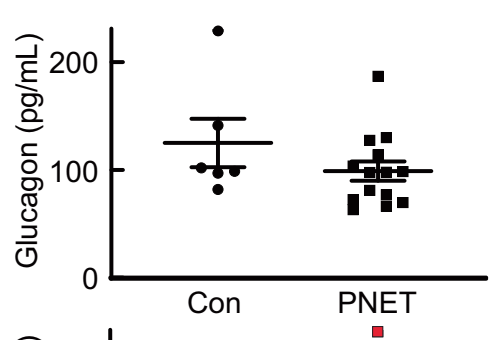

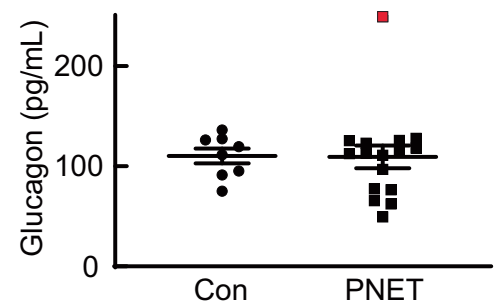

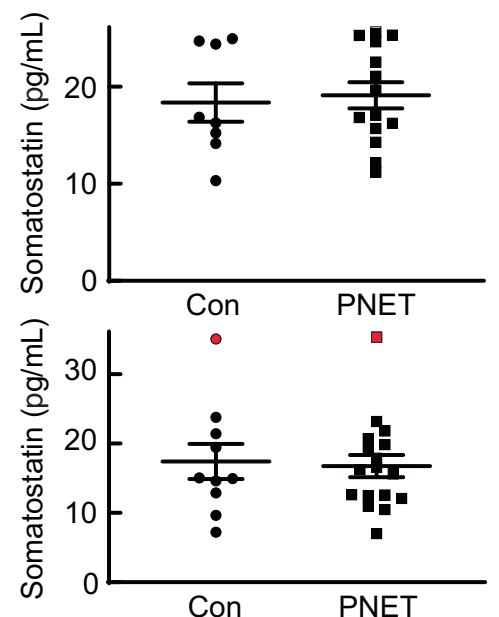

Fig. 3 Cdk5 induces both functional and non-functional PanNETs. A Immunostains of pancreas from control and three representative INSp25OE animals. ELISA assays of hormone levels in blood plasma of female (B) and male (C) control (Con; females $n=6$, males $n=8)$ and tumor-bearing (PNET; females $n=14$, males $n=21$ ) mice. Error bars are SEM; red points illustrate samples with levels that are two SD above the average for controls.

contained one animal with somatostatin levels elevated greater than two SD above the mean of the control population, again falling within the natural expected Gaussian curve.

Additionally, we tested the plasma of seven females and seven males lacking large tumor masses but found to possess abnormal islets by histopathological evaluation (data not shown). Insulin was elevated in the plasma of 1 of the 7 additional females. This female also exhibited elevation in somatostatin. One separate female possessed elevated plasma glucagon levels. In males, 2 of the 7 exhibited elevated plasma glucagon, one exhibited elevated plasma insulin, and one exhibited elevated somatostatin. Although immunostaining evaluation identified tumors that were positive for both insulin and glucagon, no animals were found to possess elevation of serum levels of both hormones. One animal, of 45 examined, exhibited elevations in both insulin and somatostatin. Collectively these data demonstrate that $48 \%$ of 


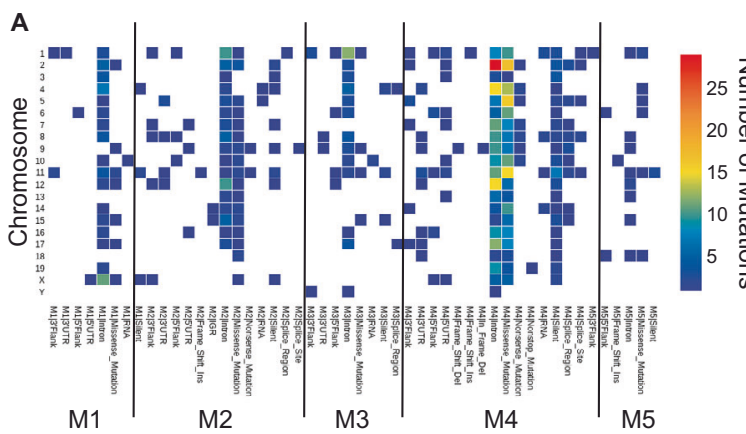

C
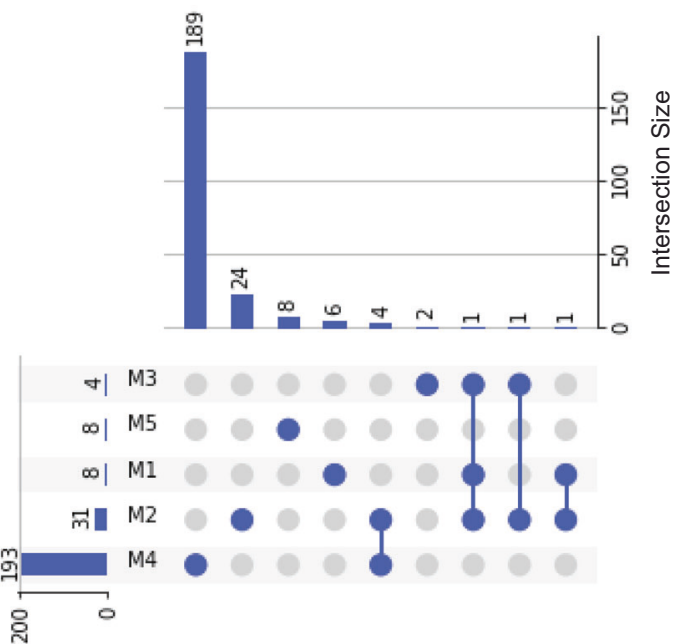

B

500

$=$ SNP

ran

$300+$ DEL

$200-1 D N P$

100

0

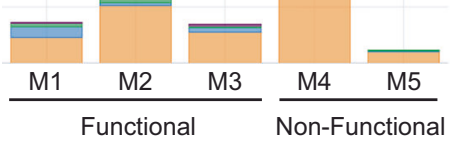

D

Sample $\mid$ Mutations

\begin{tabular}{c|l}
\hline M1 & \\
\hline M2 & $\begin{array}{l}\text { ATAD2, PACSIN3, ZC3H18, } \\
\text { PLXND1, NOTCH3, FBN2 }\end{array}$ \\
\hline M3 & \\
\hline M4 & OTOF, SPPL3, PTPRQ, SCUBE1, \\
& TKTL2, MAP4K4, PML, RBM15B, \\
& MIB2, GALNT6, CCDC30, ROBO2, \\
& SON, OSBPL8, NAT6, KPNA2, \\
& TRNAU1AP, HEATR5A, NFKB2, \\
& MMP24, TBC1D9B, DOCK2, MDN1, \\
& SPG7, GIGYF1, MLXIPL, RYR2, \\
& COL18A1, NUDCD1, NT5DC1, \\
& IPO4, ELK1, HMCN2, ANKRD17, \\
& TTN, SH2D3C, ZFHX3, SH3BGR, \\
& KIF26A, OBSCN \\
\hline M5 & TP53, NCOR2
\end{tabular}

Fig. 4 INS-p25OE tumors possess mutations found in human PanNETs. A Heat map of number of total mutations in each INS-p25OE PanNET by classification per chromosome. B Type of mutations present in each INS-p25OE PanNET. (SNP single nucleotide polymorphism, INS insertion, DEL deletion, DNP double nucleotide polymorphism). C Waterfall plot of overlapping exonal mutations among INS-p25OE PanNETs. D Table of genes in which mutations in INS-p25OE PanNETs overlap with mutations in human PanNETs.

PanNETs generated in the INS-p25OE model are potential insulinomas and $52 \%$ do not produce elevations in the serum hormones analyzed and are likely non-functional.

Expression of insulin in all tumors and elevation of circulating insulin levels in $48 \%$ of PanNET animals suggested approximately half of the tumors were functional insulinomas. However, no premature death was observed in the animals as would be expected from severe hypoglycemia due to overexpression of insulin. To investigate more thoroughly, we tested blood glucose levels in several female and male animals following a 4-6 h fasting window. Surprisingly, only $7 \%$ of females (1 of 14) and $23 \%$ of males ( 4 of 17), showed depressed circulating glucose levels under these conditions compared to transgene $(-)$ littermate controls (Supplementary Fig. S3A, B). Because mild insulinemia might take longer to affect glucose levels, we then tested both 4 and $8 \mathrm{~h}$ fasting windows in a small set of tumor-bearing females and found that only $17 \%$ (1 of 6) exhibited hypoglycemia even after $8 \mathrm{~h}$ without food (Supplementary Fig. S3C). Collectively, these data point toward $52-83 \%$ of tumors generated from this model being non-functional.

Mutation of the menin gene is the most common genetic alteration found in human PanNETs, although the prognostic implications of this mutation are a point of contention. To begin to determine if menin and Cdk5 tumorigenic pathways overlap, we analyzed the presence of menin, Cdk5, p35, and downstream components of the menin pathway in PanNETs from the MEN ${ }^{+-}$ model (MEN) and the INS-p25OE model (Supplementary Fig. S4A). As expected, levels of menin were reduced in $\mathrm{MEN}^{+/-}$tumors. Analysis of the downstream targets of menin, $\mathrm{p} 18^{\mathrm{Ink} 4 \mathrm{C}}$ and $\mathrm{p} 27^{\mathrm{KIP} 1}$, also revealed decreased expression in $\mathrm{MEN}^{+/-}$tumors compared to INS-p25OE tumors. This comparison suggests that aberrant activation of the Cdk5 pathway does not lead to inhibition of genes targeted by menin.

Levels of Cdk5 and p35 were also reduced in $\mathrm{MEN}^{+/-}$tumors, suggesting that PanNETs arising from loss of function mutations in menin are not driven by aberrant activation of $\mathrm{Cdk} 5$. To explore this observation further, we interrogated phosphorylation levels of three proteins previously identified as downstream targets of aberrant Cdk5 in thyroid neuroendocrine tumors: phospho-Ser18 histone H1.5, Ser988 RBL1, and Ser391 SUV3H1 [15]. Interestingly, each of these markers was highly phosphorylated in INS-p25OE tumors. In contrast, these signals were almost completely absent in normal islets as well as MEN ${ }^{+/-}$tumors, further supporting that loss of menin does not lead to aberrant activation of Cdk5 as a part of its tumorigenic process (Supplementary Fig. S4B). Together, these data indicate that menin and Cdk5 pathways constitute separate and independent tumorigenic pathways.

While these studies show that tumors retain dependence upon Cdk5 activity for sustained growth, the variability in age of onset combined with $75 \%$ penetrance by 12 months of age (Supplementary Fig. S2) raises the possibility that additional alterations occur and facilitate tumor formation. To investigate this further, we performed whole exome sequencing on five INS-p25OE PanNETs; three functional and two non-functional tumors. Interestingly, high heterogeneity was observed in the genetic landscape of these tumors as is also found in human tumors (Fig. 4). Several classes of mutations were observed throughout multiple chromosomes including alterations in introns, exons, $3^{\prime}$ UTRs, and $5^{\prime}$ UTRs (Fig. 4A). Single nucleotide polymorphisms (SNPs) were the most common type of alteration detected (Fig. 
4B). Examination of mutations from translated regions revealed very little overlap among samples (Fig. 4C). Although mutations in identical genes among INS-p25OE tumors were rare, alterations in genes encoding regulatory subunits of the PIK3 pathway were found in three of the five samples (60\% of INS-p25OE tumors). Mutations in the catalytic subunit of PIK3 are known to be enriched in human PanNETs [13]. This finding prompted a full comparison with sequencing datasets from human PanNETs, which revealed that 48 genes with mutations in coding regions in INS-p25OE tumors are also mutated in a published set of 98 human PanNETs [42] (Fig. 4D and Supplemental Table S2).

To further understand the molecular changes that lead to tumor development in the INS-p25OE model, we performed mRNA sequencing on six INS-p25OE PanNETs, three functional and three non-functional tumors, and compared levels of gene expression to that observed in normal mouse islets (Fig. 5A). Interestingly, higher heterogeneity was observed in the non-functional group than in the functional group (Fig. 5B). Comparing the total tumor group to normal islets, we found that 796 genes were upregulated while 533 genes were downregulated (Fig. 5C). Of note, genes such as BRCA2, STAT4 and TOP2A were dysregulated, similar to previous observations from human PanNETs (Fig. 5D) [43, 44]. Ingenuity Pathway Analysis revealed upregulation of four pathways that relate to cell cycle regulation, one pathway that involves DNA repair, one that is important for vascularization, and three that are linked to collagen and extracellular matrix regulation (Fig. $5 \mathrm{E})$.

To further investigate the validity of the INS-p25OE model for the study of human disease, we performed comparisons of gene expression data from INS-p25OE tumors with previously acquired datasets from human PanNETs and human PDACs (Supplementary Fig. S5) [45-47]. In order to compare between species and platforms, we normalized these count datasets to Reads Per Kilobase Million (RPKM) values and log transformation was applied on RPKM values [48, 49]. Principle component analysis of resulting data revealed high overall similarity between the INS-p25OE tumors and human PanNETs (Supplementary Fig. S5B). Dimension 1 , containing the highest variability $(20.2 \%)$, showed segregation of human PDACs from all three PanNET samples, supporting previous work demonstrating that PDACs and PanNETs have significantly distinct molecular profiles [50]. In dimension 2, containing the second highest variability $(6.1 \%)$, the two human PanNET datasets separate from one another indicating minor differences may arise during processing of independent sample sets. However, the percentage of variability in this dimension is low and the INS-p25OE tumors overlap entirely with one of the human PanNET sets. Gene cluster analysis further demonstrates that the INS-p25OE model shares a high degree of similarity with human PanNETs and less similarity with human PDACs.

To more thoroughly compare menin-deficient mouse models to the INS-p25OE model, beyond the signaling proteins interrogated in Supplementary Fig. S4, we compared differential gene expression data from both. To our knowledge, RNAseq data has not be collected for menin-deficient mouse models. We obtained microarray data previously analyzed to identify differentially expressed genes between conditional menin $^{-/-}$islets and normal islets [51]. This data uncovered 110 genes that were downregulated, along with 74 genes that were upregulated, in conditional menin ${ }^{-1-}$ islets (Supplementary Fig. S6). Crossreferencing these genes in our RNAseq data, used to identify differentially expressed genes between INS-p25OE tumors and normal islets, revealed that only $2 \%$ (4 of 184) of these genes exhibited similar qualitative changes (down- or upregulated), $2 \%$ (4 of 184) exhibited opposite qualitative changes, and 14\% ( 25 of 184) were not detected. Of those not detected, $92 \%$ (23 of 25) were also not detected in the human datasets analyzed in Supplementary Fig. S5. The remaining $82 \%$ of genes that were altered in menin $^{-/-}$samples, relative to normal islets, were unchanged in INS-p25OE samples, relative to normal islets. This data further confirms the utility of the INS-p25OE mouse model as a distinct entity from existing menin-deficient models.

Although the INS-p25OE model generates genetically (Fig. 4) and phenotypically (Fig. 3 and Supplementary Fig. S3) heterogenous tumors as is observed in human patients, heterogenous models require large cohort sizes to identify responses in preclinical trials. In addition, the primary model requires 6-12 months to form tumors. Therefore, we established tumor-derived allografts, from INS-p25OE primary PanNETs, as second tool that could be utilized for quick screening in a large homogenous cohort of animals. We implanted $2 \times 2 \mathrm{~mm}$ sections of tissue from a primary tumor (P0) into five recipient $\mathrm{BL} / 6$ male mice. Allograft tissue established new tumors (P1) with $100 \%$ penetrance and, on average, within 17 weeks, reducing the timeframe for development from 45 weeks in P0 mice to 17 weeks in P1 animals (Fig. $6 \mathrm{~A}-\mathrm{D})$. Furthermore, allografts were serially passaged with $100 \%$ penetrance and established 3rd generation tumors (P2), within 8 weeks (Fig. 6C, D). Allografts retained expression of the p25-GFP transgene and tumors grew 4.3-fold in a 5-week period, exhibiting very similar growth rates as primary PanNETs (Fig. 6B, E and Fig. $2 \mathrm{~J})$. Allografts also retained the well-differentiated neuroendocrine phenotype of the primary tumors, including tumor architecture and expression of ChA and insulin (Fig. 6F).

\section{DISCUSSION}

Progress in the development of therapeutics that specifically target NETs has been hampered in part by an insufficient number of animal models in which to perform preclinical experimentation. Patient-derived xenograft models, that have become a standard for pre-clinical testing in a broad range of cancer types, have proven difficult to generate for PanNETs. To date, only one is available and models functional insulinoma [52]. While PanNETs co-occur with multiple other tumor types in diverse genetically engineered mouse models, only two main types of transgenic mouse models have been generated and utilized for pre-clinical PanNET studies prior to the development of the INS-p25OE model reported here $[53,54]$. The MEN $^{+/-}$conventional knockout model develops PanNETs, as well as parathyroid and pituitary NETs, and has been utilized to explore new therapeutics such as anti-VEGF-A monoclonal antibody therapy and Pasireotide for efficacy toward PanNETs [53, 55, 56]. This model is expected to be especially relevant to the $\sim 40 \%$ of PanNET patients that possess a mutation in the gene menin. Both pan-pancreas and islet-specific conditional homozygous knockouts of the menin gene also produce PanNETs [53]. Of note, all of the PanNETs from these menin knockout models are insulinomas or gastrinomas while $~ 85 \%$ of human PanNETs are classified as non-functional $[5,53]$. Therefore, additional models would be highly beneficial.

A second conditional transgenic mouse model of PanNETs is the RIP1-Tag2 line [57]. This model was generated by cloning the large T-antigen of SV40, a known oncogenic driver, downstream of the rat insulin promoter for expression in $\beta$-islet cells. This model develops aggressive insulinomas, including both well- and poorly differentiated subsets, and has been successfully utilized to explore new therapeutics such as sunitinib and mTOR inhibitors [58-60]. Interestingly, crossing the RIP1-Tag2 mouse model into the A/J background leads to formation of tumors that do not express insulin [61]. The A/J background has a known SNP, relative to the C57BL/6 background, in the Insm 1 gene. Insm1, which encodes a transcription factor that promotes neuroendocrine differentiation and is required for insulin expression in $\beta$ cells, was implicated in the loss of insulin expression observed in the model [62]. Development of this model will undoubtedly provide insight into non-functional tumor physiology. However, these tumors are more poorly differentiated than tumors from the parent $\mathrm{C} 57 \mathrm{BL} / 6$ background and the population of human tumors to which it is relevant will need to 


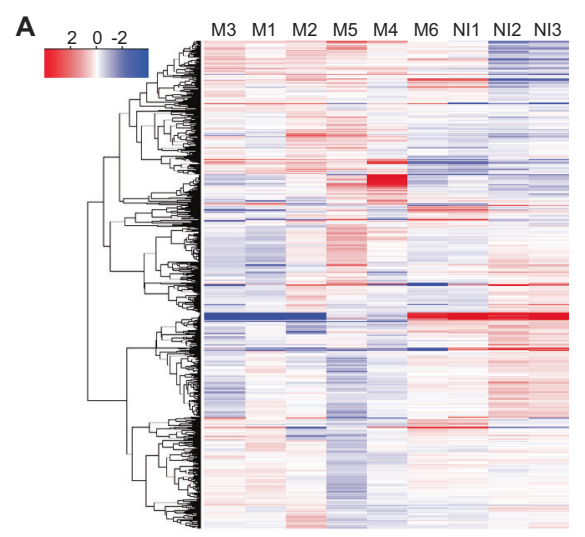

C

E

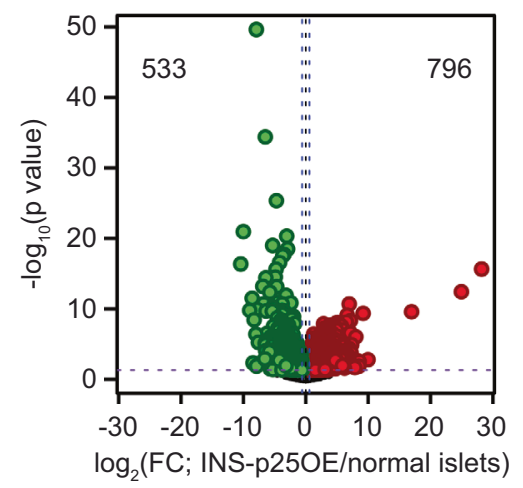

B

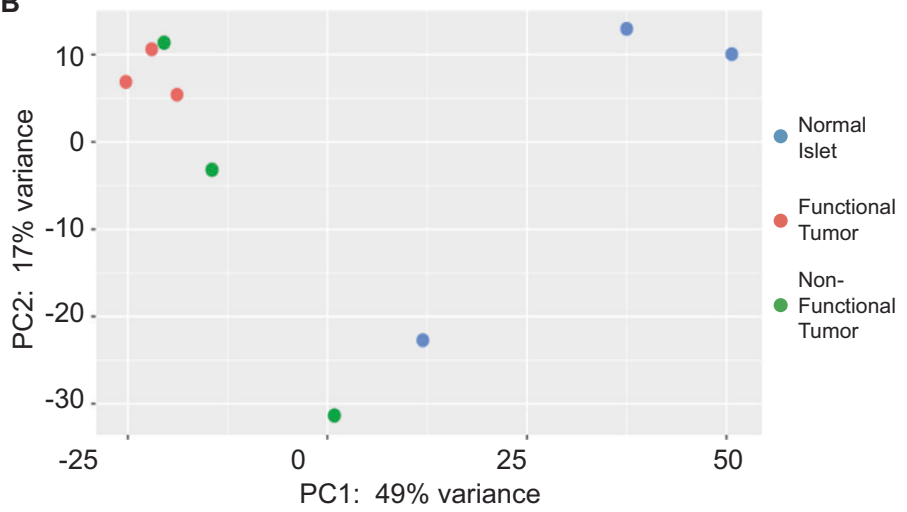

\begin{tabular}{|c|c|c|c|c|}
\hline Ingenuity Canonical Pathways & $-\log _{10}(p)$ & Ratio & z-score & Genes \\
\hline Cell Cycle Control of Chromosomal Replication & 7.11 & 0.268 & 3.873 & $\begin{array}{l}\text { CDC45, CDC6, CDC7, CDK1, CDK19, } \\
\text { CDK2, CHEK2, DBF4, DNA2, LIG1, } \\
\text { MCM7, MCM8, ORC1, PRIM2, TOP2A }\end{array}$ \\
\hline Kinetochore Metaphase Signaling Pathway & 14.9 & 0.297 & 3.657 & $\begin{array}{l}\text { AURKB, BIRC5, BUB1, BUB1B, } \\
\text { CCNB1, CDC20, CDCA8, CDK1, } \\
\text { CENPA, CENPE, CENPU, CENNPW, } \\
\text { DSN1, ESPL1, INCENP, KNL1, } \\
\text { KNTC1, MAD1L1, MASTL, MXD3, } \\
\text { NDC80, NEK2, NSL1, NUF2, } \\
\text { PPP1R3C, SKA1, SKA2, S PDL1, TTK, } \\
\text { ZWILCH }\end{array}$ \\
\hline NER Pathway & 4.22 & 0.155 & 3.606 & $\begin{array}{l}\text { CHAF1A, CHAF1B, DNA2, H4C1, } \\
\text { H4C12, H4C3, H4C6, H4C9, LIG1, } \\
\text { POLE2, POLE3, PRIM2, RFC2, RFC3, } \\
\text { RFC4, TOP2A }\end{array}$ \\
\hline GP6 Signaling Pathway & 6.28 & 0.176 & 3.13 & $\begin{array}{l}\text { COL10A1, COL11A1, COL12A1, } \\
\text { COL18A1, COL1A1, COL1A2, } \\
\text { COL3A1, COL4A1, COL5A1, COL5A2, } \\
\text { COL5A3, COL6A1, COL6A2, COL6A3, } \\
\text { COL6A4, COL8A1, GRAP2, LAMA1, } \\
\text { LAMB1, PRKCQ, RAP1B }\end{array}$ \\
\hline Intrinsic Prothrombin Activation Pathway & 2.97 & 0.19 & 2.646 & $\begin{array}{l}\text { COL10A1, COL18A1, COL1A1, } \\
\text { COL1A2, COL3A1, COL5A3, F13A1, } \\
\text { KNG1 }\end{array}$ \\
\hline Estrogen-mediated S-phase Entry & 5.52 & 0.346 & 2.333 & $\begin{array}{l}\text { CCNA2, CCNE2, CDK1, CDK2, } \\
\text { CDKN1A, E2F2, E2F7, E2F8, SKP2 }\end{array}$ \\
\hline Apelin Liver Signaling Pathway & 5.52 & 0.346 & 2.333 & $\begin{array}{l}\text { APLN, COL10A1, COL18A1, COL1A1, } \\
\text { COL1A2, COL3A1, COL5A3, MAPK10, } \\
\text { PDGFRB }\end{array}$ \\
\hline p53 Signaling & 1.97 & 0.112 & 2.333 & $\begin{array}{l}\text { BIRC5, BRCA1, CCND2, CDK2, } \\
\text { CDKN1A, CDKN2A, CHEK2, JUN, } \\
\text { PIDD1, PLAGL1, SERPINE2 }\end{array}$ \\
\hline HIF1 $\alpha$ Signaling & 2.41 & 0.098 & 2.236 & $\begin{array}{l}\text { ADM, BMP6, CDKN1A, EGF, FOXP3, } \\
\text { HMOX1, HSPA1A/HSPA1B, HSPA1B, } \\
\text { IGF1, JUN, MMP11, MMP14, MMP19, } \\
\text { MMP2, NOS2, PRKCQ, RAP1B, } \\
\text { SERPINE1, TF, VEGFD }\end{array}$ \\
\hline
\end{tabular}

Fig. 5 Cdk5 upregulates cancer-related pathways. A Heatmap of differentially expressed genes in normal mouse islets (NI1-3), functional (M1-3) and non-functional (M4-6) INS-p25OE PanNETs. B Principal component analysis of expression data. C Volcano plot of annotated genes upregulated and downregulated in INS-p25OE tumors (all 6) compared to normal mouse islets; fold change (FC). D Table of differentially expressed genes that overlap with alterations in human PanNETs; gene amplification (GA), overexpression (OE), decreased expression (DE). E Ingenuity Pathway Analysis of genes differentially expressed between INS-p25OE tumors (all 6) and normal islets. Pathways are related to cell cycle (red), DNA repair (blue), vascularization (green), and extracellular matrix (black). 
be carefully investigated as rare, poorly differentiated G3 neuroendocrine carcinomas, and relatively more abundant, welldifferentiated G3 NETs, are molecularly distinct tumor types [61, 63].

Here, we present development of a novel, dox-inducible, conditional mouse model of PanNETs in which activation of the Cdk5 pathway in $\beta$-islet cells leads to slow growing islet tumors with heterogeneous hormone production profiles, including a large subset of non-functioning, well-differentiated tumors. The utility of this model is further extended by the ability to generate multiple allograft animals from each primary PanNET. As these second-generation animals also possess a fully functional immune system, this method for generating large homogenous cohorts of immunocompetent PanNET models will be especially useful for exploration of immunotherapies, a modality whose implementation has lagged for neuroendocrine cancers [64].

Male and female cohorts were interrogated as separate groups when characterizing the INS-p25OE primary PanNET model so that differences linked to sex could potentially be uncovered. Surprisingly, although females exhibited a higher propensity for elevation of plasma insulin levels compared to males, fewer females developed hypoglycemia in response to fasting. This may be due to the fact that total insulin levels were higher in "elevated" males than "elevated" females. We have found no clinical analysis of human populations that indicate that non-functioning PanNETs are more common in one sex versus the other, although NETs in general are slightly more common in females [4].
A

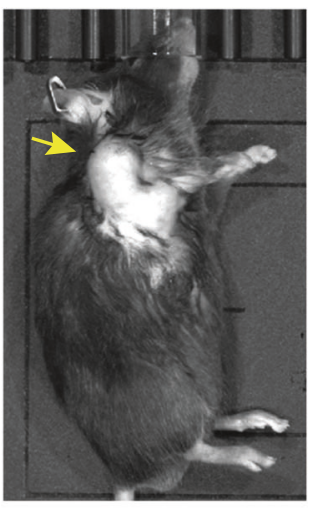

B

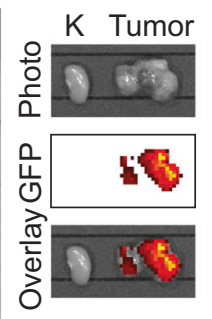

Radiance $\times 10^{8}$ $\left(\mathrm{p} / \mathrm{sec} / \mathrm{cm}^{2} / \mathrm{sr}\right)$

12345
C

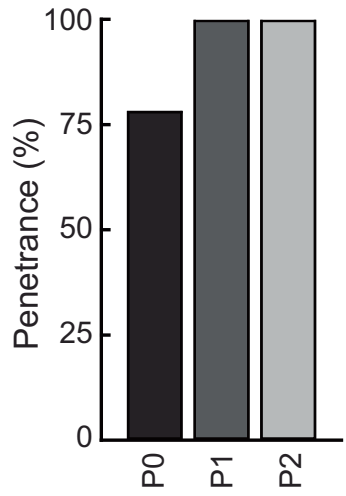

D

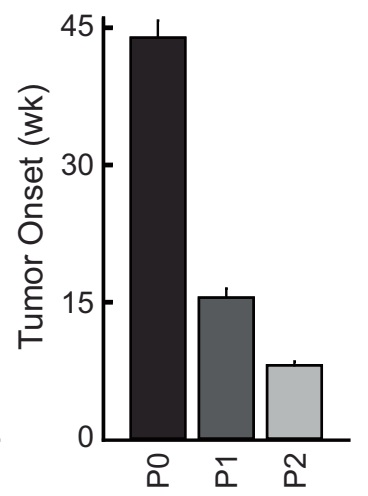

E

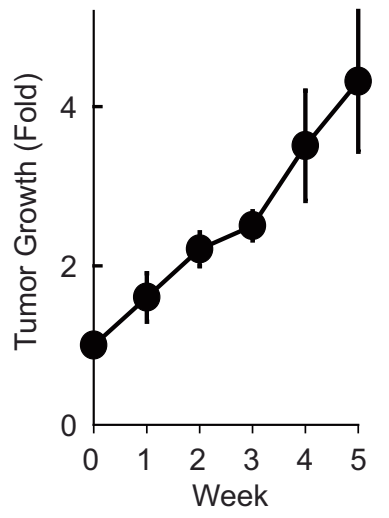

$\mathbf{F}$ P0 P1a P1b P1C

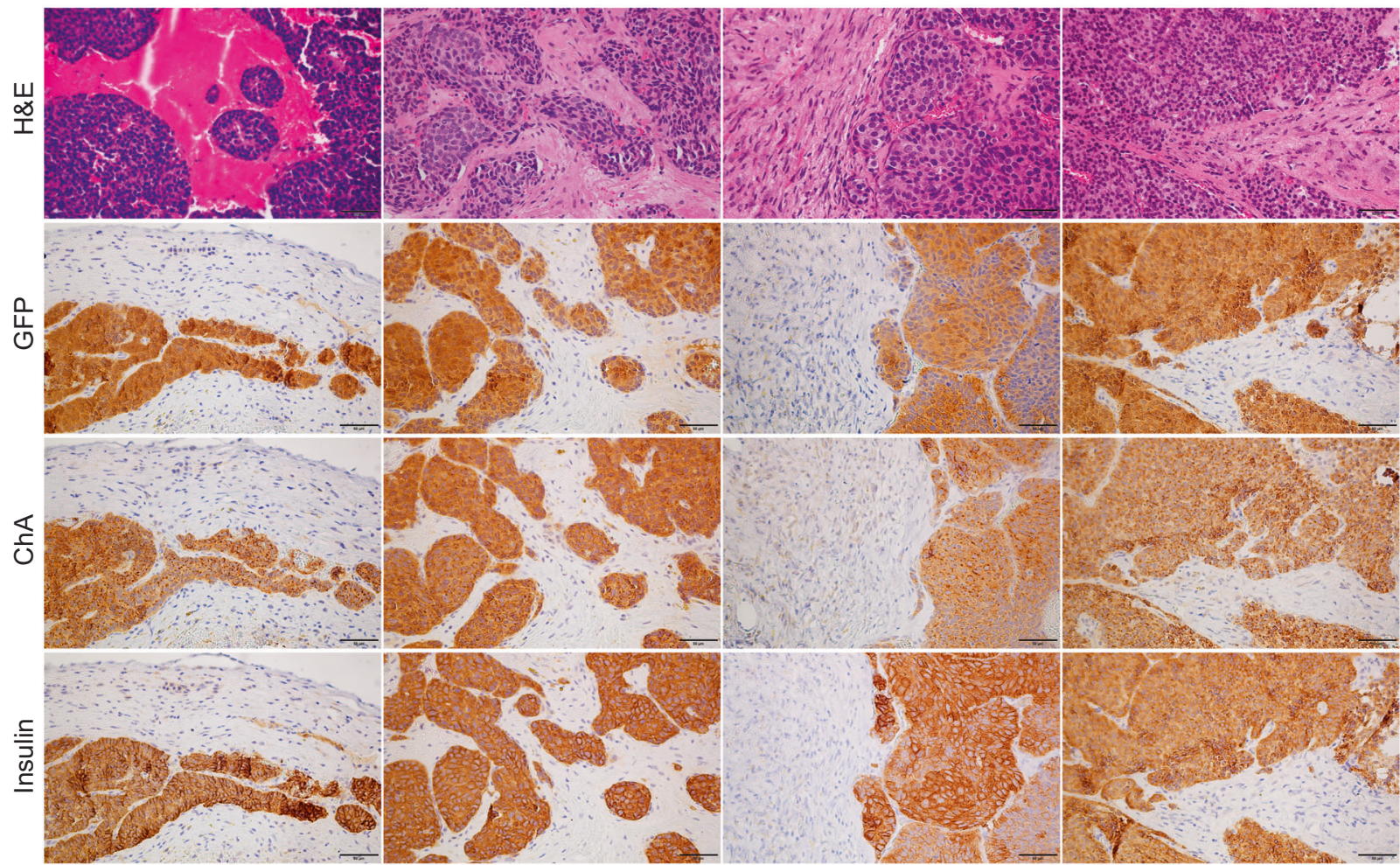

Fig. 6 INS-p25OE PanNETs form successful allografts. A Photograph from IVIS image of representative allograft tumor model; tumor marked with yellow arrow. B Photograph and fluorescence imaging from IVIS, ex vivo, of kidney (left) and tumor (right) from representative allograft model. C Penetrance in primary INS-p25OE model (P0), passage 1 models (P1), and passage 2 models (P2). D Tumor onset in the same groups as $C(\mathrm{P0}, n=21 ; \mathrm{P} 1, n=5 ; \mathrm{P} 2, n=5)$. E Fold growth of passage 1 tumors over a 5 week period starting at $\sim 100 \mathrm{~mm}^{3}($ week 0$)($ weeks $0-3, n=6$; weeks $4-5, n=5)$. F H\&E stains and immunostains of primary INS-p25OE model (PO) and three passage 1 models (P1a-c). 
The INS-p25OE model reported here is molecularly distinct from menin-deficient models and likely represents a group of human PanNETs in which mutation of the gene menin is not the key driving factor. The Menin-MLL complex targets CDKN1B and CDKN2C encoding the CDK inhibitors p27kip and p18Ink4C, respectively [65]. These inhibitors block activity of Cdk4, 6, 2, and 1. Thus, loss of function mutations in Menin can cause activation of these Cdk family members. As Cdk5 is unaffected by these inhibitors, its aberrant activation represents an alternative path to cell cycle activation. Cdk5 misregulation has been demonstrated in multiple types of human NETs $[15,18]$.

The genetic overlap between the INS-p25OE model and human PanNETs is not complete. The 48 genes, with mutations in coding regions in the INS-p25OE model, found to overlap with humans, represents $20 \%$ (48 of 244) of the total in the model. However, it is only $2 \%$ (48 of 2567 ) of the total genes mutated in the human dataset analyzed. While the difference in sample sizes (5 versus 98) precludes an absolute comparison, it suggests overlap percentages in comparable populations might be greater. In addition, even though all genetic mutations may not be identical, alterations within the same signaling pathways may be present, as found for the PIK3 pathway. Importantly, the functional transcriptome reported here points to a high degree of similarity between human PanNETs and the INS-p25OE model. Significant overlap was also observed at the functional level, as $\sim 85 \%$ of human tumors are non-functional and we observed a similar distribution of functional and non-functional tumors in the INSp25OE model. As such, this newly developed model will serve as a useful platform for molecular characterization of the population of human PanNETs in which aberrant activation of $\mathrm{Cdk} 5$ is present, as well as the development and testing of new therapeutics that target those pathways. Moreover, because this model more faithfully reflects the heterogeneity of human PanNET biology, it will facilitate development of a variety of therapeutic strategies, not limited to targeting of Cdk5.

The basis for the heterogeneity observed in this model and in humans is complex. Although random genetic mutations are known to unavoidably accumulate over multiple rounds of cell division [66] and would logically lead to differences in cellular phenotypes, this phenomenon occurs in all tumor models, not just INS-p25OE animals. Interestingly, $\beta$-cells naturally exhibit a high degree of phenotypical heterogeneity (e.g. nuclear size, granularity, polarity, hormone content) as well as functional heterogeneity (e.g. glucose responsiveness, membrane potential, insulin secretion, proliferative capacity, etc.) and only $3 \%$ of $\beta$-cells in the adult islet possess proliferative capacity $[67,68]$. Activation of Cdk5 could increase proliferation in multiple subsets of $\beta$-cells, whereas, knockdown of menin perhaps only permits growth of cells that already intrinsically possess proliferative capacitiy. Another source of heterogeneity could stem from the downstream signaling pathways targeted by Cdk5. Indeed, Cdk5 phosphorylates the methyltransferase SUV39H1, and therefore, may alter phenotypes through epigenetic modulations [15]. In addition, we postulate that Cdk5 may enhance rates of genetic mutation by targeting components of DNA replication or repair machinery. In fact, Cdk5 has been suggested to target proteins such as DNA ligase 1 and DNA polymerase epsilon [15]. In future studies, it would be interesting to analyze the mutational and transcriptional landscapes of a larger cohort of INS-p25OE tumors in order to determine if clear subgroups manifest as a result of the intrinsic heterogeneity observed in this model. These subgroups could then be compared to subgroups of human tumors, similar to previous work with the RIP1-Tag2 model [60]. Further, combining comparison of human tumors to all three types of PanNET models (menin-deficient, RIP-Tag, and INSp25OE), within the same study, would uncover valuable information regarding applicability of unique models to exact human subsets.

\section{METHODS}

\section{Human tissue collection}

Samples were collected in accordance with institutional review board (IRB) regulations under Louisiana State University IRB 5774 and University of Alabama at Birmingham IRB 300002147.

\section{Histology}

Tissues were fixed in formalin, embedded in paraffin, and sliced into $5 \mu \mathrm{m}$ sections for placement on glass slides. Samples were deparaffinized and subjected to high temperature antigen retrieval in citrate buffer $(\mathrm{pH} 6.0)$. For immunostaining, samples were permeabilized in $0.3 \%$ Triton X-100, incubated in $0.3 \%$ hydrogen peroxide, blocked with $3 \%$ normal goat serum, and then incubated overnight at $4{ }^{\circ} \mathrm{C}$ in primary antibodies. Human and mouse tissue was immunostained for Cdk5 (PhosphoSolutions 308Cdk5; 1:50) and p35/p25 (Santa Cruz sc-820; 1:50). Mouse tissue was immunostained for GFP (Cell Signaling Technology 2956; 1:200), ChA (Abcam ab15160; 1:500), insulin (Abcam ab63820; 1:2000), glucagon (Santa Cruz sc7779; 1:200), somatostatin (Abcam ab108456; 1:450). Biotinylated secondary antibodies (Pierce 31820 or $31800 ; 1: 500$ ) were applied to slides for $1 \mathrm{~h}$ at room temperature followed by $30 \mathrm{~min}$ of HRP streptavidin. Slides were then incubated with DAB Chromogen (Dako Liquid DAB + substrate K3468) and counter stained with hematoxylin. Standard procedures were used for H\&E staining. The human PanNET TMA was prepared by the UAB Research Pathology Core. Slides were immunostained as stated above. Images were deconvoluted using Fiji ImageJ. The mean intensity of a fixed region of interest for each core in the resulting $D A B$ channel was measured and then converted to optical density using the formula: OD = Log (Max intensity/mean intensity) for semi-quantitative analysis.

\section{Cell culture}

All cells were cultured in a humidified incubator at $37^{\circ} \mathrm{C}$ under $5 \% \mathrm{CO}_{2}$. Fibroblasts were grown in DMEM plus $10 \% \mathrm{FBS}$. BON and QGP cells were grown in RPMl plus $10 \% \mathrm{FBS}, 100 \mu \mathrm{g} / \mathrm{ml}$ penicillin, and $100 \mu \mathrm{g} / \mathrm{ml}$ streptomycin. NT3 and NT18 cells were cultured in RPMI 1640 GlutMAX plus $10 \% \mathrm{FCS}, 20 \mathrm{ng} / \mathrm{ml} \mathrm{EGF}, 10 \mathrm{ng} / \mathrm{ml} \mathrm{FGF2}, 100 \mu \mathrm{g} / \mathrm{ml}$ penicillin, and $100 \mu \mathrm{g} / \mathrm{ml}$ streptomycin.

\section{Cell growth assay}

Cells were seeded onto 96-well plates and allowed to adhere for $24 \mathrm{~h}$. Cells were then treated twice (day 1 and day 3) with various concentrations of inhibitor, as shown, and viability measured after 5 days by MTT assay. $\mathrm{IC}_{50}$ values were determined by 4-parameter logistic regression.

\section{INS-p250E animal model}

All animal work was performed in accordance with the Animal Welfare Act and the Guide for the Care and Use of Laboratory Animals under UTSW and UAB Institutional Animal Care and Use Committee approved protocols. Bi-transgenic INS-p25OE animals were generated from crossing of the tetOp-p25GFP strain (The Jackson Laboratory stock \# 005706) with the Ins2-rtTA strain (Provided by Dr. Alvin C. Powers at Vanderbilt; available from The Jackson Laboratory stock \# 008250). Breeders and pups were maintained in the absence of doxycycline to allow for normal development of offspring prior to transgene induction. Upon weaning, at 3-4 weeks of age, offspring were administered $1 \mathrm{mg} / \mathrm{L}$ doxycycline via drinking water to induce transgene expression in bi-transgenic animals. Bi-transgenic animals were co-housed with transgene negative littermates. Transgene negative littermates were used as normal controls. All mice were maintained in the $\mathrm{C} 57 \mathrm{BL} / 6$ background. Animals were euthanized by $\mathrm{CO}_{2}$ administration and cardiac perfusion.

\section{MRI}

MRI was performed with a Bruker Biospec 9.4 Tesla instrument using Paravision 5.1 software (Bruker Biospin, Billerica, MA). A Bruker 72 mm ID volume coil was used for excitation and a custom $24 \mathrm{~mm}$ surface coil for signal reception (Doty Scientific Inc., Columbia, SC). Mice were anesthetized with isoflurane gas and respiration observed with a MRI-compatible physiological monitoring system (SA Instruments Inc., Stony Brook, NY). Animals were imaged in supine position on a Bruker animal bed system with circulating heated water to maintain body temperature. A 2D T2weighted RARE sequence was used for imaging of the abdomen. The following imaging parameters were used: TR/TE $=2000 / 25 \mathrm{~ms}$, echo spacing $=12.5 \mathrm{~ms}, \mathrm{ETL}=4,2$ averages, 29 contiguous axial slices with 
$1 \mathrm{~mm}$ thickness, $\mathrm{FOV}=30 \times 30 \mathrm{~mm}$ and matrix $=300 \times 300$ for an in-plane resolution of $100 \mu \mathrm{m}$. Prospective respiratory gating was used to minimize motion artifacts. Tumors volumes were quantitated using ImageJ software.

\section{Immunoblot}

Cells were lysed in $1 \%$ SDS plus $50 \mathrm{mM} \mathrm{NaF}$. Samples were sonicated briefly, spun at 20,000 g for $5 \mathrm{~min}$, and supernatant combined with Laemmli buffer for analysis by SDS-PAGE followed by transfer to PVDF for immunoblotting. Tumors were crushed while frozen and then processed using the same protocol. Immunoblotting was performed using antibodies for Cdk5 (Rockland 200-301-163; 1:1000), p35 (Santa Cruz sc-820; 1:300), GFP (Cell Signaling Technology 2956; 1:2000), SSTR2 (Santa Cruz sc-365502; 1:500), Menin (Santa Cruz sc-374371; 1:250), p18Ink4c (Invitrogen 393400; 1:500), and p27Kip1 (Cell Signaling Technology 2552; 1:1000), pS18H1.5 (Bibb Lab; [15] 1:1000), H1.5 (Santa Cruz sc-247158; 1:1000), pS988RBL1 (Bibb Lab; [15] 1:1000), RBL1 (Santa Cruz sc-318; 1:500), pS392-SUV39H1 (Bibb Lab; [15] 1:300), SUV39H1 (Sigma S8316; 1:500), and actin (Invitrogen AM4302; 1:5000). Revert 700 Total Protein Stain (LICOR 926-11011) was used per manufacturer's protocol.

\section{Whole exome sequencing}

The analysis of raw WES data was performed using MoCaSeq pipeline (source code: https://github.com/roland-rad-lab/MoCaSeq). The pipeline was set up using the docker container and Ubuntu Linux. Specifically, the raw reads were trimmed aligned to the mouse reference genome $\mathrm{GRCm} 38$. p6 using Trimmomatic 0.38 and BWA-MEM 0.7.17, respectively. For further post-processing, Picard 2.20.0 and GATK 4.1.0.0 were used. For the loss of heterozygosity (LOH) analyses from WES data, somatic SNP calling was performed using Mutect2. To avoid ambiguous SNP positions resulting from mis-mapping, only reads with a mapping quality of 60 were kept in LOH analyses. For CNV calling, CopywriteR 2.6.1.216 was used, which extracts DNA copy number information from targeted sequencing by utilizing off-target reads. Finally, the downstream analysis and visualization were done using custom Python (v.3.8) and Shell scripting. DNA sequencing data generated from INS-p25OE tumors and first reported in this manuscript were deposited in NCBI SRA under PRJNA736305. Data from mice were compared to human data deposited with the European Genome-Phenome Archive under EGAD00001002684.

\section{RNASeq analysis}

RNA was isolated from tissue using RNeasy Plus Mini Kit (Qiagen 74134). RNA was transcribed to CDNA using NEBNext Ultra ${ }^{\text {TM }}$ RNA Library Prep Kit for Illumina (NEB E7530). RNA sequencing was performed using single-end 75 bp reads on an Illumina NextSeq500. The RAW sequences were trimmed using Trimmomatic 0.38 and low-quality reads were removed. The quantification of the expression of transcripts of preprocessed sequences was using salmon 1.4 .0 and $\mathrm{mm} 10$ mouse reference genome. The resulting quant (transcript abundance estimates) values were utilized for the differential expression analysis. Differential gene expression analysis was done using DESeq2 and for downstream analysis and visualization python (v.3.8) and Bash scripting were used. RNA sequencing data generated from INS-p25OE tumors and first reported in this manuscript were deposited in NCBI GEO under GSE176448. Human data for comparison to the INS-p25OE model was obtained from GSE116356, GSE79668, and GSE98894. All data were normalized RPKM and log transformed prior to comparison.

\section{Allograft models}

Primary tumors were removed from INS-p25OE mice and diced into $\sim 2 \times$ $2 \mathrm{~mm}$ sections. These sections were implanted subcutaneously into both the right and left flanks of C57BL/6 P1 (passage 1) recipient mice by trocar. Tumor size was monitored by measurement with calipers. P2 mice were generated by passaging $\mathrm{P} 1$ tumors into a second generation of C57BL/6 recipient mice.

\section{Statistical analysis}

All sample sizes represent biological replicates, not technical replicates. For cell viability assays, ANOVA with Tukey's multiple comparisons test was used in combination with Brown-Forsythe test for variance. For animals, comparisons between two groups were performed using a two-tailed Student's $t$-test; comparisons between three groups were performed using the Holm-Sidak method for one-way ANOVA if normality and equal variance tests passed; if normality or equal variance tests failed, comparisons between three groups were performed using Dunn's method for one-way ANOVA on Ranks. Shapiro-Wilk tests were used to determine normality and BrownForsythe tests were used to determine equal variance. All individual animals were randomly assigned to groups. Sample sizes are provided within figure legends or in results. $\left({ }^{*} p<0.05,{ }^{* *} p<0.01,{ }^{* * *} p<0.001\right)$.

\section{REFERENCES}

1. Ehehalt F, Saeger HD, Schmidt CM, Grutzmann R. Neuroendocrine tumors of the pancreas. Oncologist. 2009;14:456-67.

2. de Wilde RF, Edil BH, Hruban RH, Maitra A. Well-differentiated pancreatic neuroendocrine tumors: from genetics to therapy. Nat Rev Gastroenterol Hepatol. 2012;9:199-208.

3. Krampitz GW, Norton JA. Pancreatic neuroendocrine tumors. Curr Probl Surg. 2013;50:509-45.

4. Dasari A, Shen C, Halperin D, Zhao B, Zhou S, Xu Y, et al. Trends in the incidence, prevalence, and survival outcomes in patients with neuroendocrine tumors in the United States. JAMA Oncol. 2017;3:1335-42.

5. Rindi G, Wiedenmann B. Neuroendocrine neoplasms of the gut and pancreas: new insights. Nat Rev Endocrinol. 2011;8:54-64.

6. Chen H, Hardacre JM, Uzar A, Cameron JL, Choti MA. Isolated liver metastases from neuroendocrine tumors: does resection prolong survival? J Am Coll Surg. 1998;187:88-92. discussion 92-83.

7. Chen H, Sippel RS, O'Dorisio MS, Vinik Al, Lloyd RV, Pacak K, et al. The North American Neuroendocrine Tumor Society consensus guideline for the diagnosis and management of neuroendocrine tumors: pheochromocytoma, paraganglioma, and medullary thyroid cancer. Pancreas. 2010;39:775-83.

8. Oronsky B, Ma PC, Morgensztern D, Carter CA. Nothing But NET: a review of neuroendocrine tumors and carcinomas. Neoplasia. 2017;19:991-1002.

9. Neychev V, Kebebew E. Management options for advanced low or intermediate grade gastroenteropancreatic neuroendocrine tumors: review of recent literature. Int J Surg Oncol. 2017;2017:6424812.

10. Oberg K. Management of neuroendocrine tumours. Ann Oncol. 2004;15: iv293-298.

11. Corbo V, Dalai I, Scardoni M, Barbi S, Beghelli S, Bersani S, et al. MEN1 in pancreatic endocrine tumors: analysis of gene and protein status in 169 sporadic neoplasms reveals alterations in the vast majority of cases. Endocr Relat Cancer. 2010;17:771-83.

12. Jiao $Y$, Shi C, Edil BH, de Wilde RF, Klimstra DS, Maitra A, et al. DAXX/ATRX, MEN1, and $\mathrm{mTOR}$ pathway genes are frequently altered in pancreatic neuroendocrine tumors. Science. 2011:331:1199-203.

13. Mafficini A, Scarpa A. Genomic landscape of pancreatic neuroendocrine tumours: the International Cancer Genome Consortium. J Endocrinol. 2018;236:R161-R167.

14. Capdevila J, Casanovas O, Salazar R, Castellano D, Segura A, Fuster P, et al. Translational research in neuroendocrine tumors: pitfalls and opportunities. Oncogene. 2017;36:1899-907.

15. Carter AM, Tan C, Pozo K, Telange R, Molinaro R, Guo A, et al. Phosphoproteinbased biomarkers as predictors for cancer therapy. Proc Natl Acad Sci USA. 2020;117:18401-11.

16. Lin H, Chen MC, Chiu CY, Song YM, Lin SY. Cdk5 regulates STAT3 activation and cell proliferation in medullary thyroid carcinoma cells. J Biol Chem. 2007;282:2776-84.

17. Pozo K, Castro-Rivera E, Tan C, Plattner F, Schwach G, Siegl V, et al. The role of Cdk5 in neuroendocrine thyroid cancer. Cancer Cell. 2013;24:499-511.

18. Pozo K, Bibb JA. The Emerging Role of Cdk5 in Cancer. Trends Cancer 2016;2:606-18

19. Asghar U, Witkiewicz AK, Turner NC, Knudsen ES. The history and future of targeting cyclin-dependent kinases in cancer therapy. Nat Rev Drug Discov. 2015;14:130-46.

20. Dhariwala FA, Rajadhyaksha MS. An unusual member of the Cdk family: Cdk5. Cell Mol Neurobiol. 2008;28:351-69.

21. Malumbres M, Barbacid M. Mammalian cyclin-dependent kinases. Trends Biochem Sci. 2005;30:630-41.

22. Angelo M, Plattner F, Giese KP. Cyclin-dependent kinase 5 in synaptic plasticity, learning and memory. J Neurochem. 2006;99:353-70.

23. Tsai LH, Delalle I, Caviness VS Jr, Chae T, Harlow E. p35 is a neural-specific regulatory subunit of cyclin-dependent kinase 5. Nature. 1994;371:419-23.

24. Lee MS, Kwon YT, Li M, Peng J, Friedlander RM, Tsai LH. Neurotoxicity induces cleavage of p35 to p25 by calpain. Nature. 2000;405:360-4.

25. Patrick GN, Zukerberg L, Nikolic M, de la Monte S, Dikkes P, Tsai LH. Conversion of p35 to p25 deregulates Cdk5 activity and promotes neurodegeneration. Nature. 1999:402:615-22.

26. Daval M, Gurlo T, Costes S, Huang CJ, Butler PC. Cyclin-dependent kinase 5 promotes pancreatic beta-cell survival via Fak-Akt signaling pathways. Diabetes. 2011;60:1186-97. 
27. Lee HY, Jung $H$, Jang $I H$, Suh PG, Ryu SH. Cdk5 phosphorylates PLD2 to mediate EGF-dependent insulin secretion. Cell Signal. 2008;20:1787-94.

28. Lilja L, Yang SN, Webb DL, Juntti-Berggren L, Berggren PO, Bark C. Cyclindependent kinase 5 promotes insulin exocytosis. J Biol Chem. 2001;276:34199-205.

29. Ubeda M, Kemp DM, Habener JF. Glucose-induced expression of the cyclindependent protein kinase 5 activator p35 involved in Alzheimer's disease regulates insulin gene transcription in pancreatic beta-cells. Endocrinology. 2004; 145:3023-31.

30. Wei FY, Nagashima K, Ohshima T, Saheki Y, Lu YF, Matsushita M, et al. Cdk5dependent regulation of glucose-stimulated insulin secretion. Nat Med. 2005;11:1104-8.

31. Demelash A, Rudrabhatla $P$, Pant $H C$, Wang $X$, Amin ND, McWhite $C D$, et al. Achaete-scute homologue-1 (ASH1) stimulates migration of lung cancer cells through Cdk5/p35 pathway. Mol Biol Cell. 2012;23:2856-66.

32. Eggers JP, Grandgenett PM, Collisson EC, Lewallen ME, Tremayne J, Singh PK, et al. Cyclin-dependent kinase 5 is amplified and overexpressed in pancreatic cancer and activated by mutant K-Ras. Clin Cancer Res. 2011;17:6140-50.

33. Goodyear S, Sharma MC. Roscovitine regulates invasive breast cancer cell (MDAMB231) proliferation and survival through cell cycle regulatory protein cdk5. Exp Mol Pathol. 2007;82:25-32.

34. Liu R, Tian B, Gearing M, Hunter S, Ye K, Mao Z. Cdk5-mediated regulation of the PIKE-A-Akt pathway and glioblastoma cell invasion. Proc Natl Acad Sci USA. 2008;105:7570-5.

35. Strock CJ, Park Jl, Nakakura EK, Bova GS, Isaacs JT, Ball DW, et al. Cyclindependent kinase 5 activity controls cell motility and metastatic potential of prostate cancer cells. Cancer Res. 2006;66:7509-15.

36. Zhuang $\mathrm{K}$, Zhang J, Xiong M, Wang $\mathrm{X}$, Luo $\mathrm{X}$, Han L, et al. CDK5 functions as a tumor promoter in human colorectal cancer via modulating the ERK5-AP-1 axis. Cell Death Dis. 2016;7:e2415.

37. Benten D, Behrang Y, Unrau L, Weissmann V, Wolters-Eisfeld G, BurdakRothkamm S, et al. Establishment of the First Well-differentiated Human Pancreatic Neuroendocrine Tumor Model. Mol Cancer Res. 2018;16:496-507.

38. Chu Q, Wang L, Zhang J, Wang W, Wang Y. CDK5 positively regulates Notch1 signaling in pancreatic cancer cells by phosphorylation. Cancer Med. 2021;10:3689-99.

39. Kour S, Rana S, Contreras Jl, King HM, Robb CM, Sonawane YA, et al. CDK5 inhibitor downregulates MCl-1 and sensitizes pancreatic cancer cell lines to navitoclax. Mol Pharm. 2019;96:419-29.

40. Milo-Landesman D, Surana M, Berkovich I, Compagni A, Christofori G, Fleischer N, et al. Correction of hyperglycemia in diabetic mice transplanted with reversibly immortalized pancreatic beta cells controlled by the tet-on regulatory system. Cell Transpl. 2001;10:645-50.

41. Cruz JC, Tseng HC, Goldman JA, Shih H, Tsai LH. Aberrant Cdk5 activation by p25 triggers pathological events leading to neurodegeneration and neurofibrillary tangles. Neuron. 2003;40:471-83.

42. Scarpa A, Chang DK, Nones K, Corbo V, Patch AM, Bailey P, et al. Whole-genome landscape of pancreatic neuroendocrine tumours. Nature. 2017;543:65-71.

43. Scott AT, Weitz M, Breheny PJ, Ear PH, Darbro B, Brown BJ, et al. Gene expression signatures identify novel therapeutics for metastatic pancreatic neuroendocrine tumors. Clin Cancer Res. 2020;26:2011-21.

44. Wong HL, Yang KC, Shen Y, Zhao, EY, Loree JM, Kennecke HF, et al. Molecular characterization of metastatic pancreatic neuroendocrine tumors (PNETs) using whole-genome and transcriptome sequencing. Cold Spring Harb Mol Case Stud. 2018:4:a002329. https://doi.org/10.1101/mcs.a002329.

45. Alvarez MJ, Subramaniam PS, Tang LH, Grunn A, Aburi M, Rieckhof G, et al. A precision oncology approach to the pharmacological targeting of mechanistic dependencies in neuroendocrine tumors. Nat Genet. 2018;50:979-89.

46. Cejas $P$, Drier $Y$, Dreijerink KMA, Brosens LAA, Deshpande V, Epstein $C B$, et al. Enhancer signatures stratify and predict outcomes of non-functional pancreatic neuroendocrine tumors. Nat Med. 2019;25:1260-5.

47. Zhao L, Zhao H, Yan H. Gene expression profiling of 1200 pancreatic ductal adenocarcinoma reveals novel subtypes. BMC Cancer. 2018;18:603.

48. Lin S, Lin Y, Nery JR, Urich MA, Breschi A, Davis CA, et al. Comparison of the transcriptional landscapes between human and mouse tissues. Proc Natl Acad Sci USA. 2014:111:17224-9.

49. Zhao S, Ye Z, Stanton R. Misuse of RPKM or TPM normalization when comparing across samples and sequencing protocols. RNA. 2020;26:903-9.

50. Starzynska T, Karczmarski J, Paziewska A, Kulecka M, Kusnierz K, Zeber-Lubecka N. et al. Differences between well-differentiated neuroendocrine tumors and ductal adenocarcinomas of the pancreas assessed by multi-omics profiling. Int J Mol Sci. 2020;21:4470 https://doi.org/10.3390/ijms21124470.

51. Scacheri PC, Davis S, Odom DT, Crawford GE, Perkins S, Halawi MJ, et al. Genomewide analysis of menin binding provides insights into MEN1 tumorigenesis. PLoS Genet. 2006;2:e51.
52. Chamberlain CE, German MS, Yang K, Wang J, VanBrocklin H, Regan M, et al. A Patient-derived Xenograft Model of Pancreatic Neuroendocrine Tumors Identifies Sapanisertib as a Possible New Treatment for Everolimus-resistant Tumors. Mol Cancer Ther. 2018;17:2702-9.

53. Agarwal SK. Exploring the tumors of multiple endocrine neoplasia type 1 in mouse models for basic and preclinical studies. Int J Endocr Oncol. 2014;1:153-61.

54. Ney A, Canciani G, Hsuan JJ, Pereira SP. Modelling Pancreatic Neuroendocrine Cancer: From Bench Side to Clinic. Cancers (Basel). 2020;12:3170. https://doi.org/ 10.3390/cancers12113170.

55. Korsisaari N, Ross J, Wu X, Kowanetz M, Pal N, Hall L, et al. Blocking vascular endothelial growth factor- $A$ inhibits the growth of pituitary adenomas and lowers serum prolactin level in a mouse model of multiple endocrine neoplasia type 1. Clin Cancer Res. 2008;14:249-58.

56. Quinn TJ, Yuan Z, Adem A, Geha R, Vrikshajanani C, Koba W, et al. Pasireotide (SOM230) is effective for the treatment of pancreatic neuroendocrine tumors (PNETs) in a multiple endocrine neoplasia type 1 (MEN1) conditional knockout mouse model. Surgery. 2012;152:1068-77.

57. Hanahan D. Heritable formation of pancreatic beta-cell tumours in transgenic mice expressing recombinant insulin/simian virus 40 oncogenes. Nature. 1985;315:115-22.

58. Chiu CW, Nozawa H, Hanahan D. Survival benefit with proapoptotic molecular and pathologic responses from dual targeting of mammalian target of rapamycin and epidermal growth factor receptor in a preclinical model of pancreatic neuroendocrine carcinogenesis. J Clin Oncol. 2010;28:4425-33.

59. Pietras K, Hanahan D. A multitargeted, metronomic, and maximum-tolerated dose "chemo-switch" regimen is antiangiogenic, producing objective responses and survival benefit in a mouse model of cancer. J Clin Oncol. 2005;23:939-52.

60. Sadanandam A, Wullschleger S, Lyssiotis CA, Grotzinger C, Barbi S, Bersani S, et al. A cross-species analysis in pancreatic neuroendocrine tumors reveals molecular subtypes with distinctive clinical, metastatic, developmental, and metabolic characteristics. Cancer Disco. 2015;5:1296-313.

61. Kobayashi S, Contractor T, Vosburgh E, Du YN, Tang LH, Clausen R, et al. Alleles of Insm1 determine whether RIP1-Tag2 mice produce insulinomas or nonfunctioning pancreatic neuroendocrine tumors. Oncogenesis. 2019;8:16.

62. Lan MS, Breslin MB. Structure, expression, and biological function of INSM1 transcription factor in neuroendocrine differentiation. FASEB J. 2009;23:2024-33.

63. Zhang J, Francois R, lyer R, Seshadri M, Zajac-Kaye M, Hochwald SN. Current understanding of the molecular biology of pancreatic neuroendocrine tumors. J Natl Cancer Inst. 2013;105:1005-17.

64. Maggio I, Manuzzi L, Lamberti G, Ricci AD, Tober N, Campana D. Landscape and future perspectives of immunotherapy in neuroendocrine neoplasia. Cancers. Cancers (Basel). 2020;12:832. https://doi.org/10.3390/cancers12040832.

65. Hughes CM, Rozenblatt-Rosen O, Milne TA, Copeland TD, Levine SS, Lee JC, et al. Menin associates with a trithorax family histone methyltransferase complex and with the hoxc8 locus. Mol Cell. 2004;13:587-97.

66. Tomasetti C, Li L, Vogelstein B. Stem cell divisions, somatic mutations, cancer etiology, and cancer prevention. Science. 2017;355:1330-4.

67. Bader E, Migliorini A, Gegg M, Moruzzi N, Gerdes J, Roscioni SS, et al. Identification of proliferative and mature beta-cells in the islets of Langerhans. Nature. 2016:535:430-4.

68. Benninger RKP, Hodson DJ. New understanding of beta-cell heterogeneity and in situ islet function. Diabetes. 2018:67:537-47.

\section{ACKNOWLEDGEMENTS}

Ins2-rtTA mice were kindly provided by Dr. Alvin C. Powers (Vanderbilt University). PanNETs from the $\mathrm{MEN}^{+/-}$mouse model (18-22 months old mice) were kindly provided by Vaishali Parekh of Dr. Sunita K. Agarwal's lab (NIH/NIDDK). Human PDAC cell lines were kindly provided by Upender Manne. We thank the Pathology Core at UAB for TMA production, the UAB Small Animal Imaging Facility for MRI on mice, and the Heflin Center for Genomic Science at UAB for WES and RNAseq. We thank Boehringer-Ingelheim and Frank Gillardon for providing Indo A. This research was further supported by core capabilities provided by the O'Neal Comprehensive Cancer Center.

\section{AUTHOR CONTRIBUTIONS}

$A M C$ and JAB conceptualized the study. AMC, BH, CT, RG, and WH performed immunostaining. AMC performed biochemistry, immunoblots, ELISA assays, analysis of MRIs, quantitation of immunostaining, and data interpretation. FV performed cell growth assays. RT maintained the mouse colony, assisted with tissue harvesting and molecular biology. AMC, TRM, CG, and JBR generated allografts. HHB harvested islets from mice. CT and EMK performed pathological assessment of tumors. JT developed 
the MRI protocol. NK and MSM performed bioinformatics analyses. PG assisted with biochemistry. AMC and JAB assembled figures and wrote the manuscript. SR, RB, HC, $J S, J B R, M S M$, and JAB supervised the study. All authors reviewed and edited the manuscript.

\section{FUNDING}

This work was supported by an American Cancer Society Postdoctoral Fellowship (AMC); an American Cancer Society Research Scholars Award (JAB); a Robert E. Reed Foundation grant (HC); NIH award K08CA234209 (JBR); NIH awards P30CA013148 and S10 OD028498-01 (UAB Preclinical Imaging Shared Facility); and $\mathrm{NCl}$ award P30CA013148 (UAB O'Neal Comprehensive Cancer Center). A portion of effort for this project was facilitated by an SDHB Pheo Para Coalition Investigator Award and $\mathrm{NIH}$ awards DA033485-01, MH083711-01, NS073855-01, and R56MH116896 (JAB). JAB is the recipient of an NETRF Accelerator award.

\section{COMPETING INTERESTS}

The authors declare no competing interests.

\section{ADDITIONAL INFORMATION}

Supplementary information The online version contains supplementary material available at https://doi.org/10.1038/s41389-021-00372-5.
Correspondence and requests for materials should be addressed to Angela M. Carter or James A. Bibb.

Reprints and permission information is available at http://www.nature.com/ reprints

Publisher's note Springer Nature remains neutral with regard to jurisdictional claims in published maps and institutional affiliations.

Open Access This article is licensed under a Creative Common cc) Attribution 4.0 International License, which permits use, sharing, adaptation, distribution and reproduction in any medium or format, as long as you give appropriate credit to the original author(s) and the source, provide a link to the Creative Commons license, and indicate if changes were made. The images or other third party material in this article are included in the article's Creative Commons license, unless indicated otherwise in a credit line to the material. If material is not included in the article's Creative Commons license and your intended use is not permitted by statutory regulation or exceeds the permitted use, you will need to obtain permission directly from the copyright holder. To view a copy of this license, visit http://creativecommons. org/licenses/by/4.0/.

(c) The Author(s) 2021 